\title{
The Composition of Cosmic Rays at the Knee
}

\author{
S.P. Swordy ${ }^{a}$ L.F. Fortson ${ }^{\text {b,a }}$ J. Hinton ${ }^{a}$ J. Hörandel ${ }^{a, j}$ \\ J. Knapp ${ }^{\text {c C.L. Pryke }}{ }^{\text {a }}$ T. Shibata ${ }^{\text {d }}$ S.P. Wakely ${ }^{a}$ Z. Cao ${ }^{\text {e }}$ \\ M. L. Cherry ${ }^{f}$ S. Coutu ${ }^{g}$ J. Cronin ${ }^{a}$ R. Engel ${ }^{\text {h }}$ J.W. Fowler ${ }^{a, i}$ \\ K.- H. Kampert ${ }^{j}$ J. Kettler ${ }^{k}$ D.B. Kieda ${ }^{\text {e }}$ J. Matthews ${ }^{f}$ \\ S. A. Minnick ${ }^{g}$ A. Moiseev ${ }^{\ell}$ D. Muller ${ }^{a}$ M. Roth $^{j}$ A. Sill ${ }^{m}$ \\ G. Spiczak ${ }^{\mathrm{h}}$ \\ ${ }^{a}$ The Enrico Fermi Institute, University of Chicago, 5640 Ellis Avenue, Chicago, \\ Illinois 60637-1433, USA \\ ${ }^{\mathrm{b}}$ Dept. of Astronomy, Adler Planetarium and Astronomy Museum, Chicago, \\ Illinois 60605, USA \\ ${ }^{\mathrm{c}}$ Dept. of Physics and Astronomy, University of Leeds, Leeds LS2 9JT, U.K. \\ ${ }^{\mathrm{d}}$ Dept. of Physics, Aoyama Gakuin University, Tokyo, 157-8572, Japan \\ ${ }^{\mathrm{e}}$ High Energy Astrophysics Institute, Dept. of Physics, University of Utah, Salt \\ Lake City, Utah 84112, USA \\ ${ }^{\mathrm{f}}$ Dept. of Physics and Astronomy, Louisiana State University, Baton Rouge, LA \\ 70803, USA \\ ${ }^{g}$ Dept. of Physics, Penn State University, University Park, PA 16802, USA \\ ${ }^{\mathrm{h}}$ Bartol Research Institute, University of Delaware, Newark, DE 19716, USA \\ i Department of Physics, Princeton University, Princeton, NJ 08544, USA \\ j Institut für Experimentelle Kernphysik, University of Karlsruhe, D-76021 \\ Karlsruhe, Germany \\ ${ }^{\mathrm{k}}$ Max-Planck-Institut für Kernphysik, Saupfercheckweg 1, D-69117 Heidelberg, \\ Germany \\ ${ }^{\ell} N A S A$, GSFC, Code 660, Greenbelt, MD 207r1, USA \\ ${ }^{\mathrm{m}}$ Dept. of Physics, Texas Tech University, Lubbock, TX 79409, USA
}

\begin{abstract}
The observation of a small change in spectral slope, or 'knee' in the fluxes of cosmic rays near energies $10^{15} \mathrm{eV}$ has caused much speculation since its discovery over 40 years ago. The origin of this feature remains unknown. A small workshop to review some modern experimental measurements of this region was held at the Adler Planetarium in Chicago, USA in June 2000. This paper summarizes the results presented at this workshop and the discussion of their interpretation in the context of hadronic models of atmospheric airshowers.
\end{abstract}

Preprint submitted to Astroparticle Physics 22 October 2018 
Key words: PACS 95.85.R. Cosmic rays, Knee, Energy spectrum, Composition, Cherenkov.

\section{Introduction}

The primary cosmic ray particles extend over at least twelve decades of energy with a corresponding decline in intensity of over thirty decades. The spectrum is remarkably featureless with little deviation from a constant power law across this large energy range. The small change in slope, from $\propto E^{-2.7}$ to $\propto E^{-3.0}$, near $10^{15} \mathrm{eV}$ is known as the 'knee' of the spectrum. The coincidence of this feature with the highest energies expected from diffusive shock acceleration in supernova remnants is intriguing [1]. It can also be attributed to contributions from nearby or recent supernova events [2]. To distinguish between these various ideas, better measurements of the elemental composition of cosmic rays at these energies are essential. The accurate determination of composition has provided some of the key advances in this field at lower energies, where direct measurements are possible with detectors above the atmosphere. For example, the realization that the observed spectral slope of cosmic rays is significantly steeper than that produced in the cosmic ray sources themselves resulted from measurements with sufficient elemental resolution to separate primary source cosmic ray elements from those produced in the interstellar medium at $10^{11}$ $\mathrm{eV}[3]$.

Here we collect together various recent measurements of cosmic rays in the 'knee' region which were discussed at a workshop held in Chicago in June 2000 (http://knee.uchicago.edu). The objective of this paper is to provide an overview of the state of experimental measurements in this field and to provide some suggestions for future improvements. The systematic limitations of the type of measurements presented here are also evaluated. In the modern era, these limitations are associated in part with the validity of the various numerical simulations used to interpret the data and the absolute energy scale for the data.

The structure of this paper basically keeps to the structure of the workshop where both experimental results and various aspects of the numerical simulations were discussed. This work is not an exhaustive summary of the field but summarizes the efforts that were represented at the workshop. There are other notable experiments in this area which were not discussed in the workshop, in particular HEGRA[4] and EAS-TOP[5]. Also, developments since the workshop are not discussed here, these are summarized in the proceedings of the 27th International Cosmic Ray Conference (2001) Hamburg[6].

We begin with a brief overview of the relative merits of the experimental 


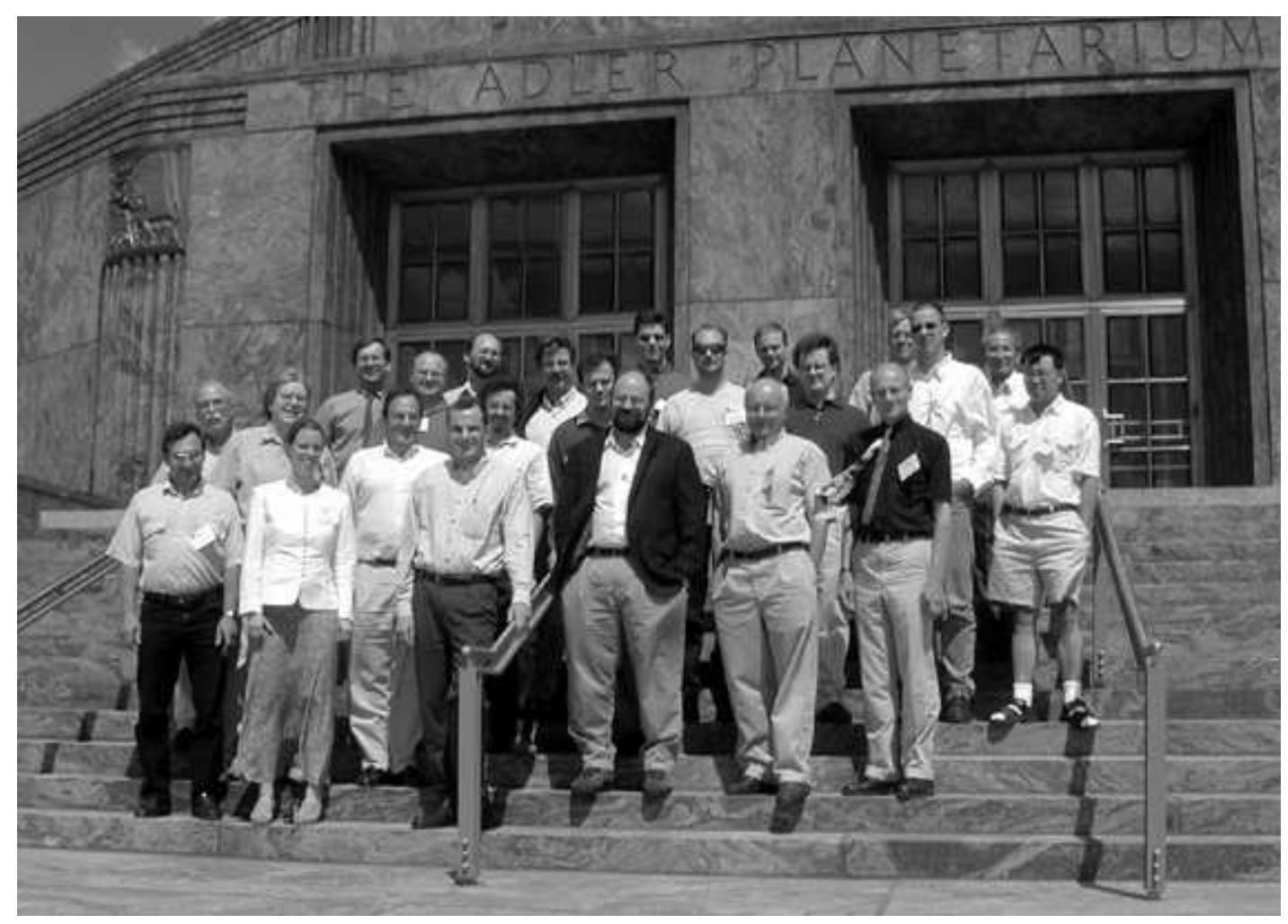

Fig. 1. Participants in the cosmic ray workshop at Adler Planetarium. (Left to Right) Moissev, Matthews, Swordy, Fortson, Sills, Cherry, Minnick, Hörandel, Kampert, Coutu, Knapp, Roth, Kieda, Wakely, Pryke, Müller, Hinton, Fowler, Cronin, Spiczak, Kettler, Shibata, Cao

techniques followed by short descriptions of direct and indirect (air shower) measurements presented at the meeting. There follows a relatively detailed discussion of air shower simulations and the physics which goes into them. We then present a simple comparison of the results of the experiments in terms of the mean logarithm of the inferred primary mass. Contemporary experiments are breaking new ground in terms of statistics and modeling, and we include some highlights of multi-species fits and multi-parameter correlation studies. Finally we draw some conclusions.

\section{Overview of Techniques}

This section seeks to summarize the main cosmic ray detection techniques and their relative merits. By no means comprehensive, the points that are touched on here were felt to be most relevant to the experiments discussed at the meeting. The experiment acronyms are defined in Section 3. 


\subsection{Direct Detection}

The detection of cosmic rays above the atmosphere is the only way to obtain direct measurements of the primary particles and their energy spectra. There are a number of techniques for direct detection. So-called "active" techniques usually involve some combination of charge detection instruments and energy measurement instruments, such as scintillator combined with a calorimeter or transition radiation detector (TRD). Here, the data can be recorded electronically in-flight. TRD's rely on the passage of a particle through the detector without a nuclear interaction while calorimeters not only require an interaction but must contain as much of the ensuing cascade as possible for accurate energy measurements. It should be noted that present TRD's saturate at about $10^{15} \mathrm{eV}$ and provide better energy measurements of high $\mathrm{Z}$ nuclei compared to protons and Helium. In contrast, calorimetric techniques can be used to obtain reasonable energy measurements of protons and Helium up to knee energies.

Nuclear emulsion is an example of a passive technique that, when interleaved with suitable converters, can simultaneously measure the primary charge and energy. These payloads must be recovered and subjected to complicated and exacting procedures to extract the energy and charge information. While each technique has its own strengths and weaknesses, the major limitation to all direct techniques to date is the inability to fly large area detectors for long periods of time. This limit is constantly being pushed as with the recent advent of Ultra-Long-Duration-Balloon flights and by constructing payloads that do not require pressurized vessels for operation. However, the nuclear emulsion experiments currently have the largest exposure and therefore the most statistically significant data at the highest energies. It is these experiments that will be discussed below.

\subsection{Indirect Detection}

Once the primary particle interacts with the Earth's atmosphere, the detection techniques indirectly measure the primary parameters through the air shower components produced in the interaction. It is then a matter of reconstructing the primary energy and nuclear charge from the air shower observables. The main difficulty with indirect techniques is that these reconstructions depend on hadronic interactions which are empirically undetermined at this time for the relevant energies and kinematical regions. However, large area, long duration experiments can be operated from the ground allowing statistically significant measurements to be made up to the highest energies in the cosmic ray spectrum. 
The different techniques can be separated into detectors that measure the particle content of the shower (KASCADE, CASA-MIA), and those that measure Cherenkov light (DICE, BLANCA, VULCAN).

The slope and normalization of the lateral distribution of an air shower component are functions of the energy and mass of the primary cosmic ray. While composition information can be obtained by lateral distribution measurements from a charged particle array (e.g. the CASA-MIA results below use the electron lateral distribution slope), in practice, it is much easier to make density measurements of the required precision when working with Cherenkov light. Because many photons are emitted by a given charged particle, and these photons are not significantly attenuated by the atmosphere, they are much more numerous than charged particles at ground level. Additionally, since the majority of the light is emitted well above the ground, and at significant angles to the shower axis, its lateral distribution at the ground is flatter than that of charged particles. For these reasons, the sensitivity and dynamic range requirements of Cherenkov detectors are not as stringent as those for charged particle detectors to probe the primary composition in a given energy range. These are the strengths behind the BLANCA and VULCAN results discussed below. However, this advantage is at least partially offset by the requirement that Cherenkov detectors can only run on dark, moonless nights.

The DICE experiment measures the angular distribution of Cherenkov light, and thus makes a somewhat direct measurement of the longitudinal profile of the air shower. This puts it a different class to all of the other experiments discussed here 1 which measure quantities which have been "integrated" over the shower development history.

The multi-component nature of air showers allows event-by-event cross-correlation measurements. This approach has been widely exploited by the KASCADE experiment, it is also possible with the DICE and BLANCA experiments which are co-located with CASA-MIA (see Section 5.3).

\section{Overview of Experiments}

Here we provide a brief description of the measurements discussed at the meeting, including direct detections by RUNJOB and JACEE and the air shower experiments KASCADE, CASA-MIA, DICE, BLANCA and SPASEVULCAN.

$\overline{1}$ With the exception of the KASCADE low energy muon tracking detectors which are not mentioned in this paper. 


\subsection{JACEE}

The Japanese-American Cooperative Emulsion Experiment (JACEE) is a series of high altitude balloon flights for direct detection of cosmic rays. The technique uses an initial charge-detecting layer for identifying the elemental charge, a target section for providing a nuclear interaction, and a calorimeter layer which collects the fraction of incident particle energy which appears as gamma-rays in the interaction $\left(\Sigma E_{\gamma}\right)$. In order to accumulate the required high energy statistics, JACEE has now flown emulsion chambers on 15 balloon flights (eight 1-2 day turnaround flights, two 5-6 day Australia-to-South America flights, and five 9-15 day Antarctic circumpolar flights [7]). All but one of these have been successfully recovered. The total accumulated exposure is $1436 \mathrm{~m}^{2} \mathrm{hrs}$. The average flight altitude ranges from 3.5 to $5.5 \mathrm{~g} \mathrm{~cm}^{2}$. A single flight typically carries 2-6 emulsion blocks, each generally $40 \times 50$ $\mathrm{cm}^{2}$. Fifty-eight emulsion blocks have been flown; fifty-two have been recovered. The data discussed here come from JACEE flights 1-12, covering the results from the first 40 emulsion blocks (cumulative exposure $644 \mathrm{~m}^{2} \mathrm{hrs}$ ). The total number of high energy events available for analysis from all flights is $\sim 2 \times 10^{4}$. Of these, $\sim 180$ have energy exceeding $100 \mathrm{TeV}$ per particle. The present analysis is based on 656 protons above $6 \mathrm{TeV}$ and 414 helium nuclei above a total energy of $8 \mathrm{TeV}$ per particle. The basic detector used in the JACEE experiments is a fine grained emulsion [8] chamber typically containing approximately a hundred track sensitive nuclear emulsion plates and a three dimensional emulsion/x-ray/lead plate calorimeter. The lower part of the chamber is the calorimeter section, consisting of $\sim 20$ layers of emulsions and xray films interleaved with up to 8.5 radiation lengths of $1-2.5 \mathrm{~mm}$ thick lead plates. The calorimeter records singly charged particle tracks with a spatial resolution in the emulsion of better than $1 \mu \mathrm{m}$ and individual photon cascades with a resolution of a few microns. High energy showers produce visible dark spots in the xray film, which are used to locate and trace the energetic cascades. On average, more than 400 events are detected per block with an optical density corresponding to a total energy in the electromagnetic shower $\Sigma E_{\gamma} \geq 1.5 \mathrm{TeV}$ for protons. In the original JACEE analyses [8-11] electron counts in the emulsion layers along the cascade were compared to a simulated shower development curve to determine the total electromagnetic energy deposited in the calorimeter. In order to speed up the analysis for the large new data sample obtained from the long duration Antarctic flights, for these flights the energy is derived by fitting the observed darkness measured in the x-ray films to electromagnetic shower development curves to determine $\Sigma E_{\gamma}[11,12]$. The results of the measured fluxes of protons and helium nuclei into the knee region are shown in Figure 2. A fit to the unbinned integral spectra gives a proton spectrum with a differential power law index $2.80 \pm 0.04$ and a helium spectrum with a differential index $2.68+0.04 /-0.06$. There is no evidence of a break in either spectrum out to at least 40-90 TeV[14]. 


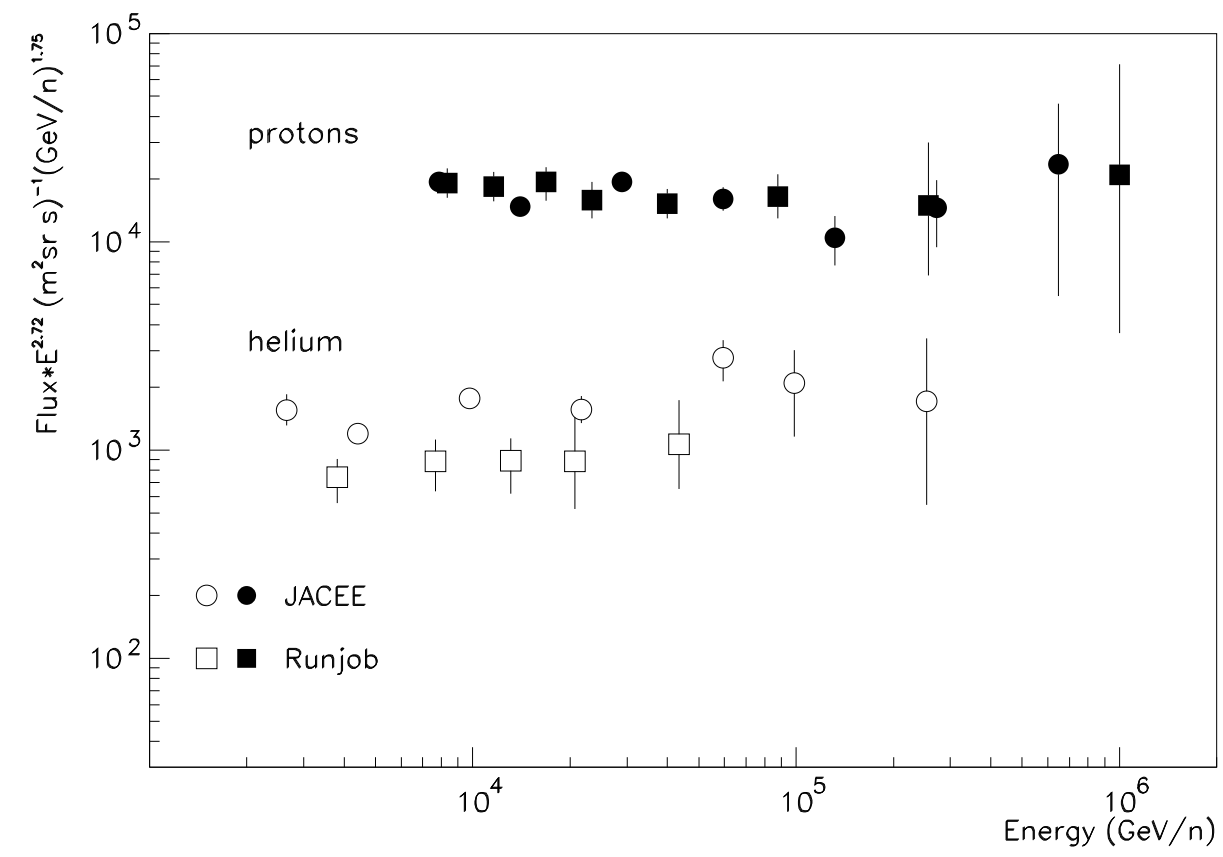

Fig. 2. Direct measurements of cosmic ray components by the JACEE [13] and RUNJOB [15] experiments

\subsection{RUNJOB}

The RUssian-Nippon Joint Balloon collaboration (RUNJOB) has been carrying out Trans-Siberian continental balloon flights since 1995, and eleven balloons have been launched from the Kamchatka peninsula, of which ten were successful. The payloads directly detect cosmic rays using a stack of passive detectors. Twenty emulsion chambers in total, each having an area of $40 \mathrm{~cm} \times 50 \mathrm{~cm}$, have been recovered near the Volga region or west side of the Ural Mountains after an exposure of approximately 150 hours. The total exposure factor amounts to $575 \mathrm{~m}^{2}$ hrs. In this work the results based on the full analysis of the '95 and '96 experiments and one quarter of the '97 experiment are given. The exposure factor used for the present data analysis amounts to $261.5 \mathrm{~m}^{2} \mathrm{hr}$. The performance of the balloon campaign and the data processing are presented elsewhere [16].

The payload mass of these flights is limited to $250 \mathrm{~kg}$ in each flight to ensure an altitude above $\sim 10 \mathrm{~g} / \mathrm{cm}^{2}$. The detector chambers consist of 4 parts: the Primary layer, Target layer, Spacer layer, and Calorimeter layer (see [15]). The primary layer is an emulsion used for charge identification, the calorimeter consists of emulsion and x-ray films. The thickness of each layer is optimized based on the experience of our analysis. There is a wide spacer between 
target module (made of lucite or stainless steel plate) and the calorimeter module, which allows a measurement of the opening angle of secondary $\gamma$-rays $\left(\pi_{0} \rightarrow 2 \gamma\right)$ produced by the nuclear interaction at the target. The spacer thickness is typically $15-20 \mathrm{~cm}$ in the shower development direction, which gives a measurement of the opening angle for individual high energy $\gamma$-rays with energy $20-50 \mathrm{TeV}$, taking the inclination effect into account. An estimate can be made of the total shower energy with $E_{\gamma} \sim 100 \mathrm{TeV}$ or more, where the electromagnetic shower maximum may not be contained in the thin calorimeter, although this does depend on the inclination of the shower. The fraction of the incoming particle energy which is released into the $\gamma$-ray component can be estimated by using cascade shower theory, which is well-established through accelerator experiments [17]. The maximum darkness of the shower in the $\mathrm{x}$-ray film is approximately proportional to the shower energy, $\Sigma E_{\gamma}$, independent of the primary particle mass. The spot darkness is measured with use of a photo-densitometer, the details of which are summarized in [18] and [19]. However, the vertical thickness of the RUNJOB chamber is as thin as 4-5 radiation lengths. A method to overcome this limitation has been discussed previously [20]. For heavy nuclei, the opening-angle method for fragments can be used. This method is summarized in detail in [21]. The results of the measured fluxes of protons and helium into the knee region are shown in Figure 2.

\subsection{KASCADE}

The KASCADE (KArlsruhe Shower Core and Array DEtector) array is an air shower detector which consists of 252 detector stations in a $200 \times 200 \mathrm{~m}^{2}$ rectangular grid located near Karlsruhe, Germany [22]. The array comprises unshielded liquid scintillation detectors $(e / \gamma$ detectors $)$ and plastic scintillators as muon detectors below $10 \mathrm{~cm}$ of lead and $4 \mathrm{~cm}$ of steel. The total sensitive areas are $490 \mathrm{~m}^{2}$ for the $e / \gamma$ detectors and $622 \mathrm{~m}^{2}$ for the muon detectors. In the center of the array a hadron calorimeter $\left(16 \times 20 \mathrm{~m}^{2}\right)$ is built up, consisting of more than 40,000 liquid ionization chambers in 8 layers [23]. In between the calorimeter a trigger layer consisting of 456 scintillation detectors of $0.456 \mathrm{~m}^{2}$ each, measures the energy deposit and arrival times of muons with E>490 MeV. Below the calorimeter an array of position sensitive multiwire proportional chambers in two layers measures EAS muons with $E_{\mu}>2.4 \mathrm{GeV}$. Various methods and results of the analysis of KASCADE data are described in detail in the proceedings of the last ICRC conference in Salt Lake City, Utah [24] or in [25], where the KASCADE contributions are sampled. The main observables of KASCADE per single shower are the so called shower sizes, i.e. total number of electrons $N_{e}$ and number of muons within the range of the core distance $40-200 \mathrm{~m}, N_{\mu}^{t r}$, local muon densities measured for the different thresholds, and number and energy sum of reconstructed hadrons at the central detector. As a phenomenological result of KASCADE it should be 
remarked that the frequency spectra of all these observables, i.e. for the different particle components, show a clear kink at the same integral event rates. This is a strong hint for an astrophysical source of the knee phenomenon based on pure experimental data, since the same intensity of the flux corresponds to equal primary energy. The multi component measurements of KASCADE, especially the investigation of the hadronic component, allow the evaluation and improvement of hadronic interaction models in kinematical and energy ranges not covered by present collider experiments [26,27].

The shower sizes $N_{e}$ and $N_{\mu}^{t r}$ are used as input parameters for a neural network analysis to reconstruct the primary energy on an event by event basis. The necessary 'a priori' information in the form of probability density distributions are found by detailed Monte Carlo simulations with large statistics. The resulting energy spectrum depends on the high energy interaction model underlying the analyses. But the spectrum shows a clear kink at $\sim 4-5 \mathrm{PeV}$ and power law dependences below and above the knee. A simultaneous fit to the $N_{e}$ and $N_{\mu}^{t r}$ size spectra is performed for the reconstruction of the primary energy spectrum. The kernel function of this fit contains the size-energy correlations for two primary masses (proton and iron) obtained by Monte Carlo simulations. This approach leads to the all particle energy spectrum as a superposition of the spectra of light and heavy particles. Besides the use of global parameters like the shower sizes, sets of different parameters (describing different shower particle components) are used for neural network and Bayesian decision analyses for showers with their axes within the central detector area. Examples of such observables are the number of reconstructed hadrons in the calorimeter $\left(E_{h}>100 \mathrm{GeV}\right)$, their reconstructed energy sum, the energy of the most energetic hadron ('leading particle' in the EAS), number of muons in the shower center $\left(E_{\mu}>2 \mathrm{GeV}\right)$, or parameters obtained by a fractal analysis of the hit pattern of muons and secondaries produced in the passive calorimeter material. The latter ones are sensitive to the structure of the shower core which is mass sensitive due to different shower developments of light and heavy particles in the atmosphere. Monte Carlo statistics limit the number of parameters which can be used for one multivariate analysis. Therefore a set of approaches using different observables are averaged in case of the actual result of the Bayesian analysis. The resulting classifications are corrected with misclassification matrices leading to relative abundances. Afterwards the results are converted into distributions of the mean logarithmic mass. The resulting all-particle energy spectrum is shown in Figure 8 [29]. Figure 11 shows the mean logarithmic mass obtained by this approach versus primary energy [28]. 
The Chicago Air Shower Array - MIchigan muon Array (CASA-MIA) detector [30] is an array of surface and underground plastic scintillators which measure the electromagnetic and muon components of air showers. The array is located on Dugway Proving Grounds, Utah, USA. The CASA-MIA data are used here to determine the probability, on an event by event basis, that a given data shower resulted from a 'light' or a 'heavy' primary. These distinctions are made by comparison to simulations of proton- or iron-induced air showers. A composition-independent measure of the energy is used to search for trends in the composition as a function of energy.

In order to study composition, a detailed fit of all events is performed after a series of standard fits determines core distance, the electron and muon sizes and other shower quantities. Both the standard and detailed fits are based on the NKG and Greisen functions; the detailed fits allow more parameters to remain free. Three parameters sensitive to composition are extracted: the density of surface particles $\rho_{e}$ and the slope $\alpha$ of the lateral distribution near the core, and the density of muons $\rho_{\mu}$ at large core distance. The parameters $\rho_{e}, \rho_{\mu}$ and $\alpha$ are tabulated for data and for simulation events. Data events are classified as 'iron-like' or 'proton-like' depending on whether these parameters most resemble iron or proton simulation events. The K Nearest Neighbor (KNN) test is employed to quantify this decision [31,32]. Each data event is placed in the three-parameter space defined by $\rho_{e}, \rho_{\mu}$ and $\alpha$. A large set of simulated iron and proton events also populate the space. Suitably normalized distances (computed in units of the variance of each parameter, with correlations included) between the datum and individual simulation events is calculated. The nuclear types of the five nearest simulation events are then tallied. The use of $\mathrm{K}=5$ is optimal for this analysis [31]. Using too few simulation points is subject to fluctuations, using too many would sample simulation points with very different primary energies. Each CASA-MIA event takes one of six possible values, corresponding to whether it has $0-5$ proton neighbors out of the five nearest neighbors examined. Using separate simulated calibration sets in place of data, it is found that more than $90 \%$ of events will have a majority of neighbors of their own species while about $50 \%$ have all nearest neighbors of their own kind. The 'proton resemblance' was normalized to the results of analyzing separate simulation calibration sets of pure iron and of pure protons. For example, near $100 \mathrm{TeV}$, the average fraction of proton nearest neighbors for a pure proton calibration sample is $68 \%$; at the same energy a pure iron sample has only $16 \%$. The average for CASA-MIA data at this energy is $51 \%$. The computed proton resemblance is approximately proportional to $\langle\log (\mathrm{A})\rangle$, the mean value of the logarithm of the atomic number of the sample [31,33]. These data are shown in Figure 11. 


\section{$3.5 D I C E$}

The Dual Imaging Cherenkov Experiment (DICE) is a ground based air shower detector which is designed to have as little reliance as possible on the details of the air shower simulations and to have the capability of comparison with existing direct measurements at $0.1 \mathrm{PeV}$ to provide an assessment of the overall systematic error. Since the method of imaging hadronic showers in Cherenkov light is a relatively recent development, we provide some more detailed description of the DICE detectors and operation. The two DICE telescopes are located at the CASA-MIA site in Dugway, Utah [30]). They each consist of a $2 \mathrm{~m}$ diameter f/1.16 spherical mirror with a focal plane detector of 256 close packed $40 \mathrm{~mm}$ hexagonal photomultipliers (PMTs) which provide $\sim 1 \mathrm{deg}$ pixels in an overall field of view $16 \mathrm{deg} \times 13.5 \mathrm{deg}$ centered about the vertical. The telescopes are on fixed mounts separated by $100 \mathrm{~m}$ (see $[34,35]$ ).

Cosmic-ray events within the field of view produce a focal plane image at the PMT cluster which corresponds to the direction and intensity of Cherenkov light coming from the air shower. When the direction of the air shower core and the distance of the shower axis from the telescopes are known, simple geometry can be used to reconstruct the amount of light received from each altitude of the shower. The amount of Cherenkov light produced is strongly correlated with the number of electrons in the shower and is used to estimate the electron size as a function of depth in the atmosphere from which the location of shower maximum can be determined. This procedure is essentially geometrical and is independent of numerical simulations except for calculations which determine the angular distribution of Cherenkov light around the shower axis. For each air shower collected a simple time coincidence is used to identify the same event in both DICE clusters and CASA-MIA. Further requirements on the correlation of the DICE images with each other and with the CASA-MIA event geometry are used to reduce the overall probability of event mismatches between the detectors to $\sim 10^{-5}$. The parameters for each shower are derived from these measured values. The accuracy of the shower core location derived by CASA is $1-3 \mathrm{~m}$ depending on the overall shower size. The measurement of the shower arrival direction is accurate to $\sim 0.4 \mathrm{deg}$ for larger showers with some degradation for lower energy events.

Previous work with DICE estimated the shower energy by a simple translation from the total amount of Cherenkov light in the image and the geometry of the shower [36]. In this work [37] a more accurate estimate of energy is derived from a combination of the amount of Cherenkov light and the location of shower maximum size $\left(X_{\max }\right.$, in atmospheric depth $\left.\mathrm{g} / \mathrm{cm}^{2}\right)$ determined by each DICE telescope. This is desirable since the lateral distribution and intensity of Cherenkov light at a given total energy depends both on the primary particle mass, hence mean $X_{\max }$, and the distance of the measurement from the shower 


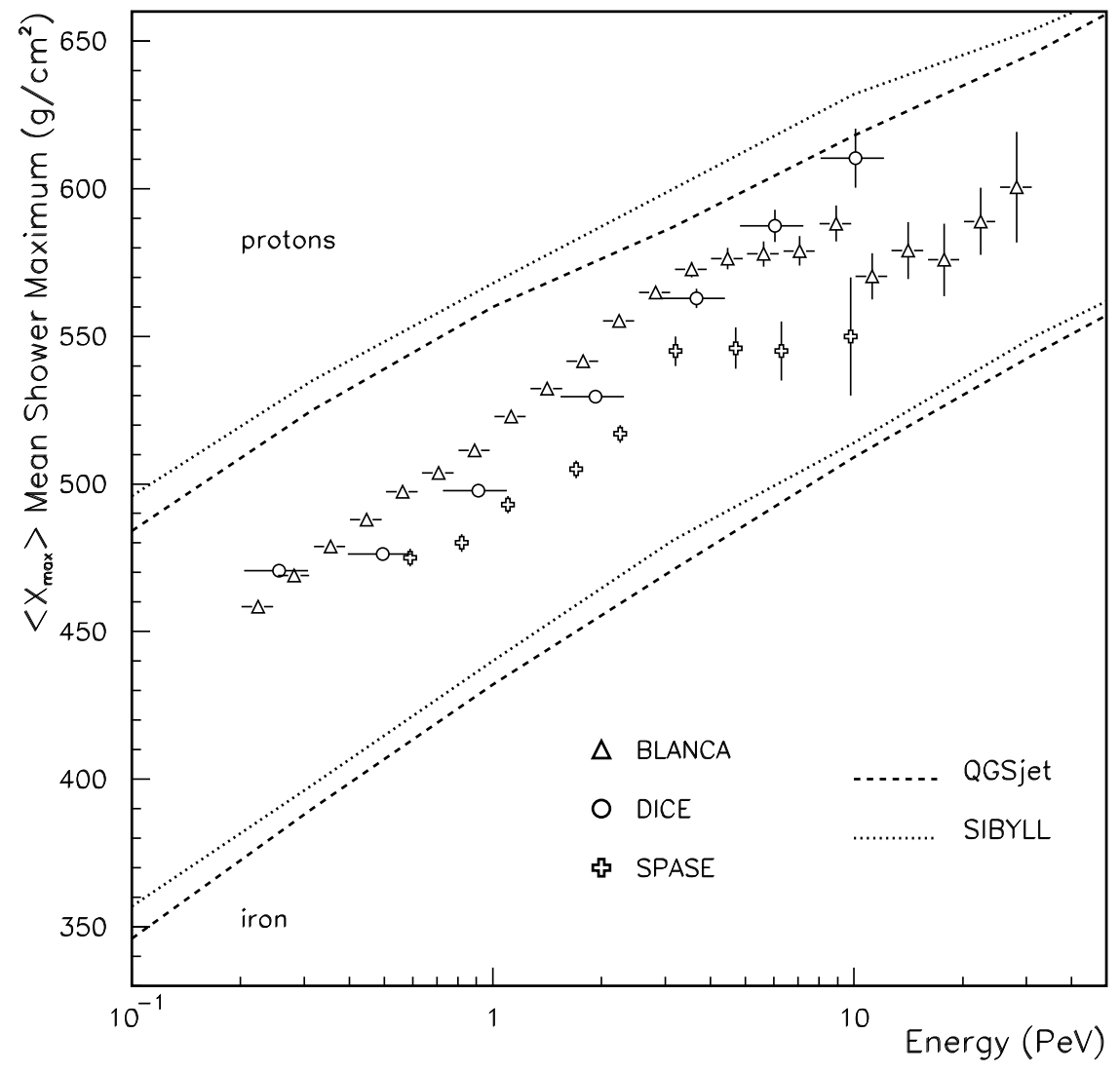

Fig. 3. Results from experiments reporting $\left\langle X_{\max }\right\rangle[39,37,53]$. Also shown are the results from model calculations discussed in text. The SIBYLL [41] calculation is from Table 1, the QGSjet is from [42].

core. A fit for the total shower energy and primary particle mass is made to the geometry, Cherenkov size and $X_{\max }$ location in the two DICE telescopes. The form of the Cherenkov size function used in these fits is derived from the results of simulations using the program CORSIKA 4.50 with the VENUS interaction model [38]. The derived $X_{\max }$ fitting function has a constant shower elongation rate parameter and assumes simple superposition for providing primary particle mass dependence.

With the acceptance cuts used during analysis the effective collection geometry is determined by the instrument Monte Carlo to be $\sim 3300 \mathrm{~m}^{2} \mathrm{sr}$, making the overall collecting power $\sim 125,000 \mathrm{~m}^{2}$ sr days. The results for the mean location of $X_{\max }$ versus energy are shown in Figure 3. 


\section{$3.6 \quad B L A N C A$}

The Broad LAteral Non-imaging Cherenkov Array (BLANCA) takes advantage of the CASA-MIA particle array installation by augmenting it with 144 angle-integrating Cherenkov detectors. Located in Dugway, Utah at an atmospheric depth of $870 \mathrm{~g} \mathrm{~cm}^{-2}$, BLANCA uses the CASA trigger to collect Cherenkov light and records the Cherenkov lateral distribution from cosmic ray events in the energy range of the knee. The CASA trigger threshold imposes an energy threshold of $\sim 100 \mathrm{TeV}$ on the Cherenkov array. However, BLANCA analysis uses events with a $200 \mathrm{TeV}$ minimum to avoid composition bias introduced from the CASA trigger.

Each BLANCA detector contains a large Winston cone [43] which concentrates the light striking an $880 \mathrm{~cm}^{2}$ entrance aperture onto a photomultiplier tube. The concentrator has a nominal half-angle of $12.5^{\circ}$ and truncated length of $60 \mathrm{~cm}$. The Winston cones were aligned vertically with $\sim 0.5^{\circ}$ accuracy. A twooutput preamplifier increases the dynamic range of the detector. The minimum detectable density of a typical BLANCA unit is approximately one blue photon per $\mathrm{cm}^{2}$.

BLANCA operated between January 1997 and May 1998. After applying data quality cuts, about 460 hours of Cherenkov observations remain corresponding to an exposure of $212,000 \mathrm{~m}^{2} \mathrm{sr}$ days. The detectors were calibrated on a nightly basis by using the circularly symmetric property of the cosmic ray air showers. This method was also verified with a portable LED flasher. The LED system was then used in the laboratory to produce single photoelectrons in a PMT with good charge resolution to obtain the absolute calibration of the BLANCA system. A full description of the detector can be found in [44].

The lateral distribution of Cherenkov light is fit to an empirical functional form to yield the normalization at $120 \mathrm{~m}\left(C_{120}\right)$ and the exponential slope between 30 and $120 \mathrm{~m}(s)$. To interpret these measurements, a large set of air shower simulations were generated with the CORSIKA Monte Carlo package. To access the systematics inherent in the choice of interaction models, four separate hadronic models were used - QGSjet, VENUS, SIBYLL and HDPM. Ten thousand events were generated for each hadronic model in four primary groups - protons, helium, nitrogen and iron [45]. The simulated air shower events were then processed through a full BLANCA detector simulation [46] to obtain "fake" data. These fake data were used to find the optimum transfer functions between the observables $C_{120}$ and $s$, and the energy and mass of the primary particle. The energy error distribution depends on the primary mass and energy. Assuming a mixed cosmic ray composition, the energy resolution for a single air shower is approximately $12 \%$ for a $200 \mathrm{TeV}$ shower, falling to $8 \%$ for energies above $5 \mathrm{PeV}$. In a similar way, a transfer function from slope, $s$, 
to $X_{\max }$ was determined and the results are plotted in Figure 3. The quantity that is of direct astrophysical interest is the mean nuclear mass of the primary. In obtaining an absolute nuclear mass from the Cherenkov observables we chose to translate directly from $\left(C_{120}, s\right)$ to $\ln (A)$ to avoid compounding the errors that would occur by using primary energy and $X_{\max }$ as intermediate quantities. For the purposes of this paper however, a translation through our $X_{\max }$ values has been done for direct comparison with other groups. The results are shown in Figure 9. Full details of the BLANCA analysis are in [39].

\subsection{SPASE/VULCAN/AMANDA}

An experiment to measure the electron, muon and air-Cherenkov components of $\sim 1 \mathrm{PeV}$ air-showers has been established at the geographic South Pole $\left(688 \mathrm{~g} / \mathrm{cm}^{2}\right)$. The experiment comprises: the SPASE-2 scintillator array [47]; the VULCAN air-Cherenkov array [48], and the deep under-ice muon detector AMANDA [49]. Simultaneous measurement of the electron size, high energy $(>500 \mathrm{GeV})$ muon content and lateral distribution of Cherenkov light should allow a composition measurement that is relatively insensitive to model assumptions.

SPASE-2 (a $1.6 \times 10^{4} \mathrm{~m}^{2}$ array of plastic scintillator modules) provides an event trigger with a threshold of $\sim 50 \mathrm{TeV}$ for proton primaries. The SPASE2 data are used to determine the shower core/direction to an rms accuracy of $4 \mathrm{~m} / 1^{\circ}$ at $1 \mathrm{PeV}$. Digitized waveforms from the nine element VULCAN array (similar in design to AIROBICC [50]) are used to reconstruct the lateral distribution of Cherenkov light. For a subset of events the shower axis passes through the AMANDA detector and the ice-Cherenkov signal can be used to estimate the $>500 \mathrm{GeV}$ muon content of the shower. The aperture of the SPASE-2/AMANDA-B10 telescope is $\approx 100 \mathrm{~m}^{2} \mathrm{sr}$.

The lateral distribution of Cherenkov light from air-showers is closely related to their longitudinal development [51] and can be used to provide an indirect measurement of the depth of shower maximum $\left(X_{\max }\right)$ and primary energy $(E)$. The MOCCA air-shower simulation [52] (coupled with the SIBYLL hadronic interaction model [41]) has been used to deduce $X_{\max }(E)$ from the Cherenkov data obtained using the VULCAN array during 1997 and 1998 (see Figure 3). The systematic uncertainties in $X_{\max } / E$ are $\approx 15 \mathrm{~g} / \mathrm{cm}^{2} / 20 \%$. This work is described in detail in [53] and [48]. A preliminary investigation of the distribution of $X_{\max }$ at around $1 \mathrm{PeV}$ suggests a ratio of (proton+helium)/all $=0.13_{-0.02}^{+0.03}$ for the MOCCA/SIBYLL-17 model [54]. However the probability of compatibility with the data is low $(2 \%)$. A much better fit is obtained by shifting the $X_{\max }$ distributions to the mean values predicted by the CORSIKA/VENUS model [55]: (proton + helium) $/$ all $=0.39_{-0.01}^{+0.03}$ with a probability 
of $40 \%$.

For the subset of SPASE-2/VULCAN/AMANDA coincidences, a measurement of muon content from AMANDA can be combined with the VULCAN energy determination to estimate primary mass. Preliminary results from this method are consistent with the SPASE-2/VULCAN $X_{\max }$ result but have limited statistics. These statistics will be increased by more than a factor of three by the addition of data from 1999 and 2000 in which the projected area of the AMANDA was increased.

In addition the SPASE-2 and VULCAN surface arrays have been useful calibration and surveying tools for the AMANDA detectors. It is anticipated that a surface air-shower detector would form a natural part of any future ice-Cherenkov neutrino observatory.

\section{Simulation Models for Air Showers}

The measurement of extensive air showers (EAS) is presently the only way to study cosmic rays with high statistics at energies near or above the knee of the all-particle spectrum. The properties of the primary cosmic rays have to be deduced from the shape and the particle content of the EAS. The energy of the primary is approximately reflected in the total number of secondaries produced and its mass can be deduced from the shower form. An iron primary e.g., consisting of 56 nucleons, would produce roughly a superposition of 56 nucleon showers of energy E/56, thus being shorter with a shower maximum higher up in the atmosphere than a proton shower of the same energy.

Since experiments at knee energies or above cannot be calibrated with a test beam the interpretation of EAS measurements is performed by comparison of the experimental data with model predictions of the shower development in the atmosphere. Therefore, unfortunately, quantitative results rely on the model assumptions and on the quality of the simulation of particle interactions and transport in the atmosphere.

While the electromagnetic interaction (responsible for electromagnetic showers, ionization, Cherenkov light production, etc.) and the weak interaction (responsible for decays of unstable secondaries) are rather well understood, the major uncertainties in Monte Carlo simulations of EAS arise from the hadronic interaction models. The development of an EAS for a given energy and primary is strongly dependent on the inelastic cross-sections $\sigma_{\text {inel }}$ of primary and secondary particles with air and on the fraction of the available energy transferred into secondary particles (usually termed inelasticity). Large cross-sections and high inelasticity produce short showers, while small 
cross-sections and low inelasticity produce long showers penetrating deep into the atmosphere. Unfortunately a systematic variation in cross-section and/or inelasticity in the model would directly translate into uncertainties of the primary mass estimates obtained from the height of the shower maximum.

The present theoretical understanding, in the form of QCD, cannot be applied to calculate the hadronic inelastic cross-section or the particle production from first principles. Therefore hadronic interaction models are usually a mixture of basic theoretical ideas and empirical parameterizations tuned to describe the experimental data at lower energies. Unfortunately none of the collider experiments register particles emitted into the extreme forward direction. These particles, being produced in collisions with rather small momentum transfer, carry most of the hadronic energy and are of the greatest importance in the development of EAS, since they transport a large energy fraction deep into the atmosphere. Moreover, since cosmic ray energies exceed those accessible by accelerators by several orders of magnitude, models have to be extrapolated well beyond the range of firm knowledge. Here one has to rely on theoretical guidelines.

\subsection{CORSIKA and the hadronic interaction models}

The most widely used air shower model is the CORSIKA program [56,57]. It is a fully 4-dimensional Monte Carlo code, simulating in great detail the evolution of EAS in the atmosphere initiated by photons, nucleons, or nuclei. Single particles are tracked, accounting for energy loss, deflection due to multiple scattering and the Earth's magnetic field, kinematically correct decays of unstable particles, and electromagnetic and hadronic interactions. CORSIKA contains various hadronic interaction models which permits a comparison and an estimate of systematic errors due to model assumptions and uncertainties in the hadronic interaction. The available models are HDPM [58,56], VENUS [59], QGSjet [60], SIBYLL [41], and DPMJET [61] and, for the energies below $\mathrm{E}_{\text {lab }}=80 \mathrm{GeV}$ per nucleon, GHEISHA [62].

VENUS, QGSjet, and DPMJET model hadronic interactions of nucleons and nuclei on the basis of the Gribov-Regge theory (GRT) [63], which most successfully describes elastic scattering and, via the optical theorem, the total hadronic cross-section as a function of energy. In GRT the observed rise of the cross-sections is a consequence of the exchange of multiple supercritical pomerons. Inelastic processes are described by cut pomerons leading to two color strings each, which fragment subsequently to color neutral hadrons. The probability of $\mathrm{n}$ exchanged and $\mathrm{m}$ cut pomerons is uniquely given by the theory. While this is common for all implementations of the GRT, they differ in the way the production and decay of the strings is realized. (A good introduc- 
tion to GRT models is given in [59].)

SIBYLL is a minijet model. It simulates a hadronic reaction as a combination of one underlying soft collision in which two strings are generated and a number of minijet pairs leading to additional pairs of strings with higher $p_{\perp}$ ends. In this model the rise of the cross-section is due to the minijet production only.

HDPM is a purely phenomenological generator which uses detailed parameterizations of $p \bar{p}$ collider data for particle production. The extension to reactions with nuclei and to energies beyond the collider energy range are somewhat arbitrary.

GRT models simulate nucleus-nucleus collisions in great detail allowing for multiple interactions of nucleons from projectile and target. The configurations of interacting projectile and target nucleons are determined with a Glaubertype geometrical picture of the nucleons in a nucleus [64]. HDPM and SIBYLL employ for nuclear projectiles the superposition model assuming an independent reaction for each of the projectile nucleon.

QGSjet, DPMJET, and SIBYLL account for minijet production, which becomes dominant at higher energies and leads to increasing cross-sections and more high- $p_{\perp}$ particles. While in QGSjet and DPMJET minijets contribute only partially to the rise of the cross-section, they are solely responsible for it in SIBYLL.

VENUS is the only model taking into account the interactions of intermediate strings and secondary hadrons with each other, leading to better agreement with final states as measured in collider experiments. In DPMJET these interactions are only implemented for secondary hadrons.

For energies below $80 \mathrm{GeV}$ GHEISHA is used, which was written around 1985 to simulate the interactions of $\mathrm{GeV}$ secondaries from $e^{+} e^{-}$collisions with typical detector materials. GHEISHA is a phenomenological model, i.e. it parameterized particle production to agree with beam test results.

Detailed comparisons of the models available in CORSIKA have been performed $[55,65]$ revealing differences on the $25 \%$ to $40 \%$ level and proving some of the models unable to describe aspects of the experimental results at all (e.g. SIBYLL and KASCADE results [66]). In the last three years some of the models were updated and new models were added to CORSIKA.

QGSjet.00 is an updated version of QGSjet with an increased Pomeron-nucleon coupling leading mainly to a cross-section with a $5 \%$ larger value at $\sqrt{s}=1800$ $\mathrm{GeV}$, which is well within the variation of the experimental results [67-69] of about $10 \%$. This was attempted to explain the trigger and hadron rates in 
the KASCADE experiment [70]. However, while the EAS data could be reproduced better by QGSjet.00 the rapidity distributions for $p-\bar{p}$ collisions were no longer reproduced by the model. Therefore it was decided to stick with the previous cross-sections.

An error in the implementation of the DPMJET cross-sections was discovered and corrected which brought the values down by about $10 \%$.

A new version of SIBYLL (2.1) [71] was produced as a response to the deficit of muons in SIBYLL 1.6. SIBYLL now allows for multiple string production in soft collisions, which yields more muons and modifies the resulting crosssections. Also better structure functions have been introduced to improve the extrapolations to high energies, and the treatment of nucleus-nucleus collisions was improved. Version 2.1 of SIBYLL is not yet officially released. The results shown here are based on a preliminary version which was available to the authors for evaluation.

The model NEXus [72] emerges as a new and more powerful program constructed by the authors of VENUS and QGSjet. NEXUS is based on GRT but includes also hard interactions and attempts to describe all types of high energy interactions in a uniform way. NEXUS version 2 is implemented in the next CORSIKA release.

URQMD [73] is an alternative to GHEISHA for low energy hadron interactions. It has been used in heavy ion collision experiments. It performs a more detailed modelling and would avoid problems with GHEISHA at a few GeV energies, but it turns out not to be free of problems either.

\subsection{Inelastic Cross-Sections}

A summary of experimental and predicted cross-sections is shown in Figure 4. Back in 1998 the variation of the p-air cross-section in experiments as well as in models amounted to about $25 \%$ at $3 \times 10^{15} \mathrm{eV}$, and to about $40 \%$ at $10^{18} \mathrm{eV}$ (shown in the left panel). For nucleus-air cross-sections the model uncertainty was only 10-15\% due to the averaging effect of many nucleons inside the nucleus. The right panel shows the situation after some of the model authors have revised their cross-sections and new theoretical predictions have been published. HDPM, VENUS and MOCCA are not shown since they are no longer supported by their respective authors.

Recent calculations of the p-air cross-sections from Frichter et al. [74] agree well with the QGSjet cross-sections. However, beyond $10^{17} \mathrm{eV}$ the predictions become slightly higher than those of QGSjet. Block et al. also [75] performed new calculations by using a QCD inspired parameterization of accelerator 

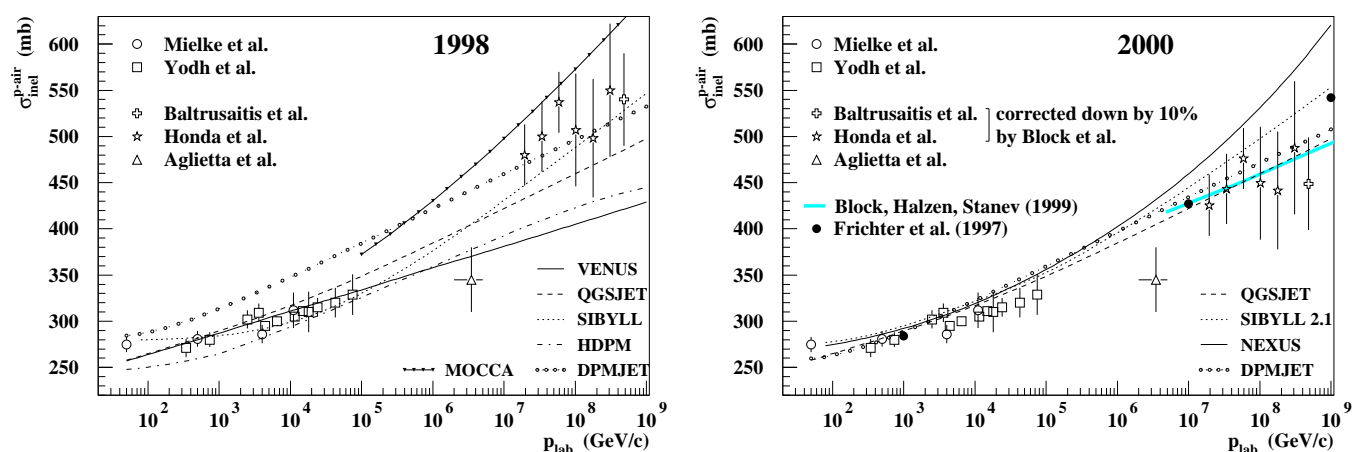

Fig. 4. Inelastic p-air cross-sections. left: Situation in 1998 right: Situation in 2000 data. They simultaneously fit the total p-p cross-sections, the ratio of real to imaginary part of the forward scattering amplitude $\rho$, and the nuclear slope parameter B. Then the p-p cross-sections were converted via Glauber theory into p-air cross-sections. Their result agrees very well with the cross-sections used in the QGSjet model.

Extracting cross-sections directly from EAS measurements is not possible. Usually the attenuation length $\Lambda$ of EAS in the atmosphere is measured which relates to the cross-sections $\sigma_{\text {inel }}^{\mathrm{p}-\text { air }}$ via $\Lambda=\mathrm{k} \cdot 14.6 \mathrm{~m}_{\mathrm{p}} / \sigma_{\text {inel }}^{\mathrm{p}-\text { air }}$, where $\mathrm{k}$ depends strongly on the average inelasticity (and the inelasticity distribution) of $\mathrm{p}$ and $\pi$ reactions and on the energy. While early and naive models used the inelasticity as one of the free parameters, in GRT models the inelasticity distribution emerges inevitably as a result of more fundamental properties of the interaction model. Usually $\mathrm{k}$ is assumed from an EAS model, which biases the final results for all the experimental cross-sections. In addition, different groups have been using different values for $\mathrm{k}$. The experimental results can be easily brought into agreement with each other and with the theoretical calculations by slightly modifying k [75]. For the AGASA and Fly's Eye data such a correction brought the experimental values in the energy range $10^{16}-10^{18} \mathrm{eV}$ down by $12-20 \%$, which now are in good agreement with the cross-sections as predicted by Block et al. or QGSjet.

As a result of various modifications the model predictions at knee energies agree now to within about $6 \%$.

\subsection{Shower Development}

The cross-sections together with the inelasticity determine the longitudinal development of an EAS, which in turn is closely related to the most important shower observables: particle number at ground, height of shower maximum and total energy in the electromagnetic component. In Figure 5 average shower curves of $10^{15} \mathrm{eV}$ showers from different models are shown. 
While for the old models the position of $\mathrm{X}_{\max }$ varied by about $7.5 \%$ for protons and $5.5 \%$ for iron showers, these number with the new models are only $1 \%$ and $0.5 \%$ respectively. Also the particle numbers at ground level agree now much better than two years ago. The variations for $\mathrm{p}$ and Fe showers were reduced from $80 \%$ and $50 \%$ to $14 \%$ and $3 \%$, respectively.

Calculations with URQMD instead of GHEISHA yield differences mainly in the low energy part of the showers. E.g. with URQMD there are about $30 \%$ more baryons in the energy range $2-10 \mathrm{GeV}$ and a factor of 2 less for $0.1-2$ $\mathrm{GeV}$ than with GHEISHA. Also, while URQMD tends to produce $10 \%$ more muons below $4 \mathrm{GeV}$ and $10 \%$ less above $4 \mathrm{GeV}$, the total number of muons stays approximately the same in both cases.

The position of the shower maximum $\mathrm{X}_{\max }$ and its variation with energy are often used to estimate the mean mass and mass composition of primary cosmic rays. In Figure 6 calculated $\mathrm{X}_{\max }$ values are shown for QGSjet, SIBYLL 1.6 and SIBYLL 2.1.

None of the models show a linear relation as is often assumed in oversimplified arguments. As compared to SIBYLL 1.6, the new SIBYLL predicts about 20 $\mathrm{g} / \mathrm{cm}^{2}$ smaller $\mathrm{X}_{\max }$ values for both proton and iron induced showers. SIBYLL 2.1 and QGSjet agree very well for iron showers and for proton showers until about $10^{18} \mathrm{eV}$. Beyond this energy QGSjet gives smaller values. In the right panel of Figure 6 fluctuations are also shown. The shaded bands indicate the 1- $\sigma$ region around the average $\mathrm{X}_{\max }$ values. Note that the fluctuations of $\mathrm{X}_{\max }$ for proton showers are of the order of 100 to $60 \mathrm{~g} / \mathrm{cm}^{2}$, i.e. not much smaller than the separation between protons and iron. These fluctuations make particle identification using $\mathrm{X}_{\max }$ for single events very difficult and require high statistics measurements to determine the average values reliably. In the case of QGSjet the differences between proton and iron showers decrease with energy, and consequently the bands for proton and iron start to overlap at
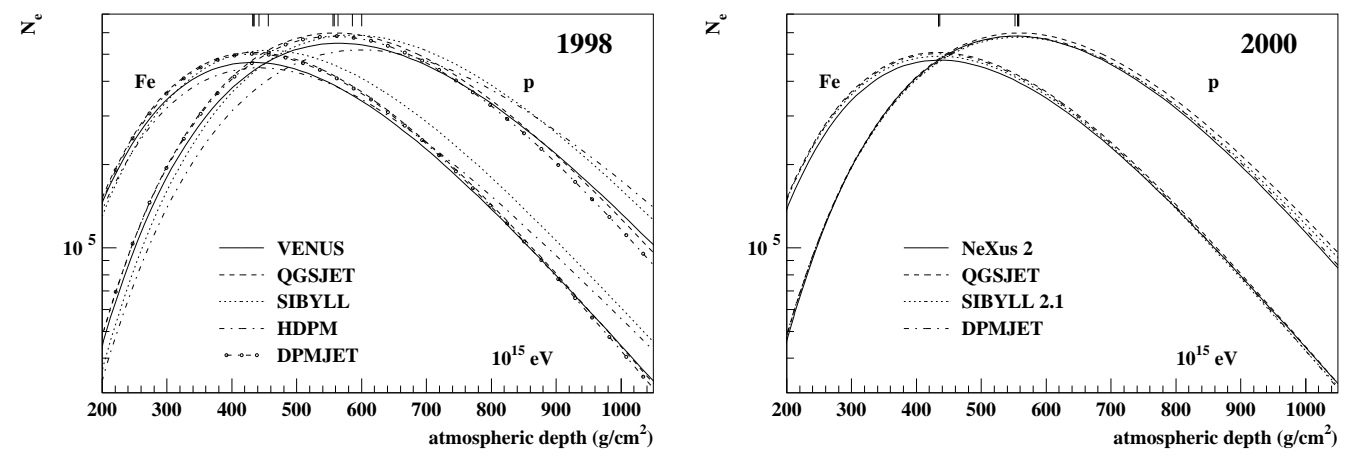

Fig. 5. Longitudinal shower development. The positions of the shower maxima are indicated at the upper rim of the picture. left: Situation in 1998 right: Situation in 2000 

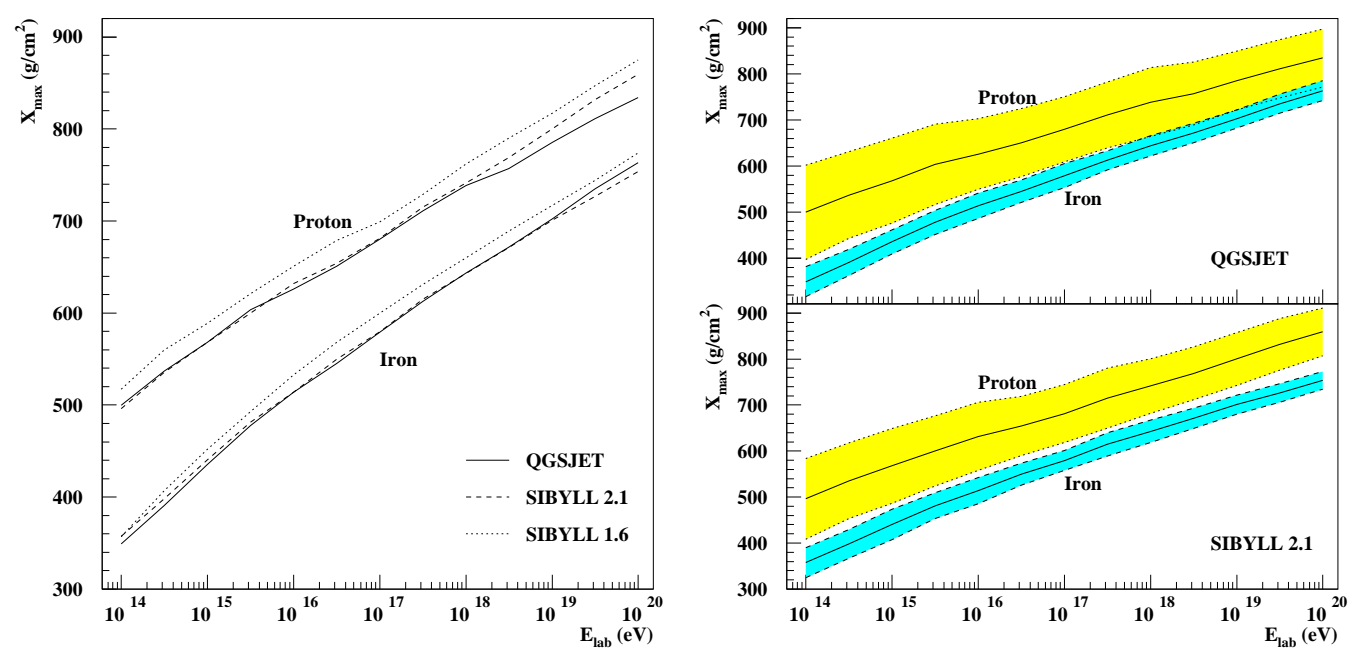

Fig. 6. Height of shower maximum $\mathrm{X}_{\max }$ as a function of energy. left: The curves for QGSjet, a preliminary version of SIBYLL 2.1 and SIBYLL 1.6 are shown. right: Average values together with the $1-\sigma$ band indicating the standard deviation of the $\mathrm{X}_{\max }$ distribution. [76]

high energies, while for SIBYLL 2.1 they remain separate. Thus according to QGSjet the separation of different masses becomes more difficult with energy than according to SIBYLL 2.1. The $\mathrm{X}_{\max }$ values are summarised in Table 1.

\subsection{Collider Results}

Another, and sometimes underestimated, source of uncertainties are the errors of the collider results which are used to tune the interaction models. There are a few instances where the inherent precision of those results is revealed.

The different inelastic $\mathrm{p}-\overline{\mathrm{p}}$ cross-sections as measured by experiments at Fermilab were already mentioned [67-69]. Their results vary from $80.0 \pm 2.2 \mathrm{mb}$ to $71.7 \pm 2.0 \mathrm{mb}$ at $\sqrt{s}=1800 \mathrm{GeV}$ or $\mathrm{E}_{\text {lab }}=1.7 \times 10^{15} \mathrm{eV}$. This corresponds to an $11 \%$ uncertainty already below the knee, and clearly much more for the highest energy cosmic rays.

A second example is the pseudorapidity distribution of secondary particles produced in $\mathrm{p}-\overline{\mathrm{p}}$ collisions. The UA5 measurements from $\sqrt{s}=200$ to 900 $\mathrm{GeV}[77]$ have been widely used to tune hadronic interaction models. GRT models always had severe difficulties reproducing the rapidity densities $\mathrm{dN} / \mathrm{d} \eta$ near $\eta=0$ and the slope in $\mathrm{dN} / \mathrm{d} \eta$ at around $\eta=4$ at the same time. However, more recent measurements by Harr et al. [78] show a clearly flatter distribution than the UA5 ones, that fit very well to the GRT predictions (see Figure 7). In the near-forward region $(\eta \approx 4)$ the differences in the particle densities amount to about $25 \%$. 


\begin{tabular}{|c|cc|cc|}
\hline \multirow{2}{*}{} & \multicolumn{2}{|c|}{ QGSjet } & \multicolumn{2}{c|}{ SIBYLL 2.1} \\
& proton & iron & proton & iron \\
\hline showers & 700 & 200 & 500 & 200 \\
\hline Energy & $\mathrm{X}_{\max }$ & $\mathrm{X}_{\max }$ & $\mathrm{X}_{\max }$ & $\mathrm{X}_{\max }$ \\
$(\mathrm{eV})$ & $\left(\mathrm{g} / \mathrm{cm}^{2}\right)$ & $\left(\mathrm{g} / \mathrm{cm}^{2}\right)$ & $\left(\mathrm{g} / \mathrm{cm}^{2}\right)$ & $\left(\mathrm{g} / \mathrm{cm}^{2}\right)$ \\
\hline \hline $10^{14}$ & $500 \pm 102$ & $349 \pm 33$ & $496 \pm 88$ & $357 \pm 32$ \\
$3.16 \times 10^{14}$ & $537 \pm 94$ & $391 \pm 29$ & $535 \pm 83$ & $398 \pm 31$ \\
$10^{15}$ & $568 \pm 92$ & $435 \pm 27$ & $568 \pm 81$ & $440 \pm 33$ \\
$3.16 \times 10^{15}$ & $603 \pm 87$ & $478 \pm 26$ & $600 \pm 76$ & $481 \pm 28$ \\
$10^{16}$ & $626 \pm 77$ & $514 \pm 28$ & $632 \pm 74$ & $514 \pm 28$ \\
$3.16 \times 10^{16}$ & $651 \pm 74$ & $545 \pm 24$ & $654 \pm 64$ & $550 \pm 24$ \\
$10^{17}$ & $680 \pm 71$ & $579 \pm 26$ & $681 \pm 63$ & $580 \pm 22$ \\
$3.16 \times 10^{17}$ & $711 \pm 71$ & $612 \pm 21$ & $715 \pm 65$ & $615 \pm 25$ \\
$10^{18}$ & $739 \pm 75$ & $644 \pm 22$ & $742 \pm 59$ & $643 \pm 25$ \\
$3.16 \times 10^{18}$ & $757 \pm 68$ & $672 \pm 21$ & $769 \pm 57$ & $671 \pm 22$ \\
$10^{19}$ & $786 \pm 64$ & $702 \pm 20$ & $800 \pm 57$ & $701 \pm 21$ \\
$3.16 \times 10^{19}$ & $811 \pm 63$ & $735 \pm 21$ & $832 \pm 56$ & $726 \pm 20$ \\
$10^{20}$ & $834 \pm 63$ & $764 \pm 22$ & $859 \pm 52$ & $753 \pm 19$ \\
\hline
\end{tabular}

Table 1

Average $\mathrm{X}_{\max }$ and standard deviation for proton and iron showers and QGSjet and SIBYLL 2.1 models. The values are determined from 200 showers per energy point for iron and from 500 to 700 showers per energy for protons by fitting the shower maximum to each individual shower and averaging over the fit results [76].

\subsection{Summary of air shower models}

In the last two years much has changed in EAS simulations. There is a clear trend of convergence between different hadronic interaction models, which, to some extent, is due to objective improvement of the physics put into the models. Presently QGSjet seems to be the best model showing a good overall agreement with a variety of experimental results. Typical differences in crosssections or inelasiticities with other upcoming models (SIBYLL 2, NEXUs) are of the order of $10 \%$. Unfortunately the number of independent models may be effectively reduced from 5 to 2 (NEXUs and SIBYLL 2), which will make it more difficult to estimate systematic uncertainties in the future, although a completely new version of DPMJET is in development[79]. Further improvement will be difficult since there is a variety of uncertainties at the $10 \%$ level in 


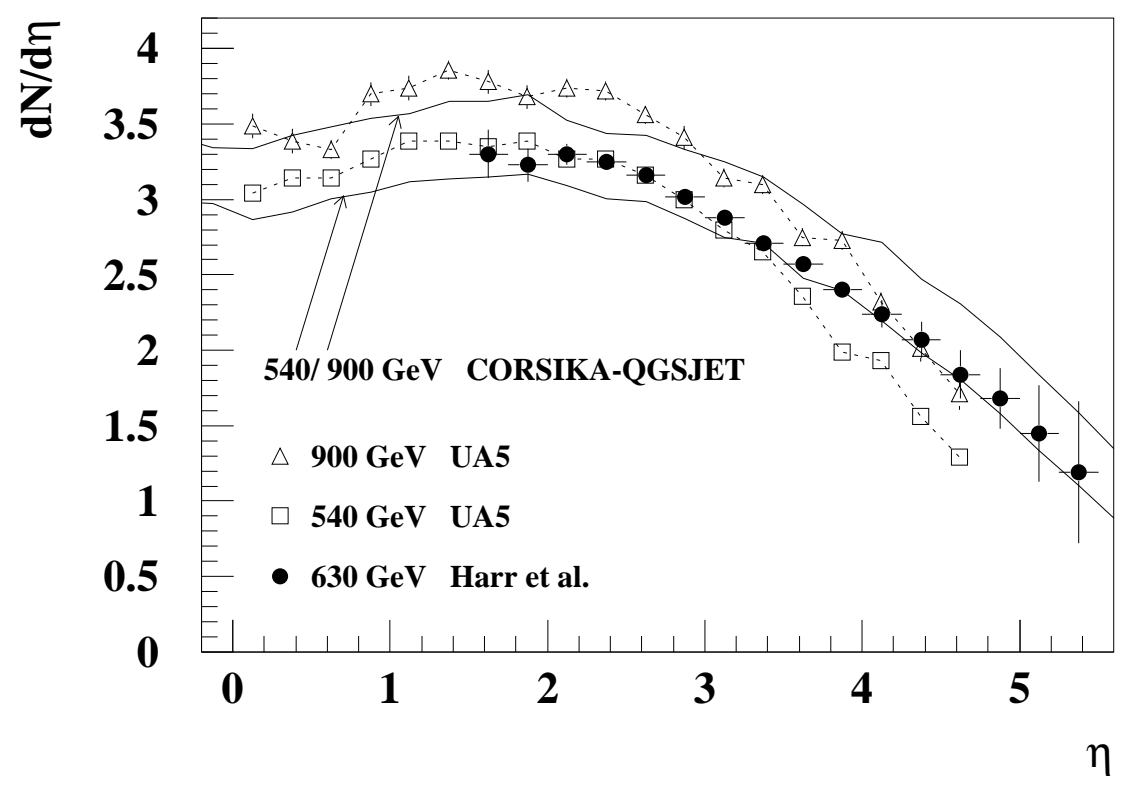

Fig. 7. Pseudorapidity distributions as measured and simulated.

collider results, in theoretical asumptions and, even larger ones, in experimental air shower results, that enter into construction of EAS models. This makes it rather unlikely that EAS model predictions will get much better in the near future. A promising source of new information on hadronic interactions in the longer term will be the experiments at LHC and the new heavy ion colliders, even though CERN decided not to pursue the proposed experiments for very small angle secondaries.

It is also unfortunate that many of the experiments investigating the composition and spectra at the knee have ceased operation (CASA-MIA-BLANCA, DICE, EAS-TOP, HEGRA-AIROBICC). Also here it will be in future more difficult to combine different aspects of the problem and to estimate the systematics. However, high statistics and good quality data are a good basis for substantial further improvement, perhaps with more model independent analysis techniques.

\section{Discussion of Results}

\subsection{Energy Spectra}

Part of the intent of the workshop, and this report, is to examine how the various experiments are consistent in the measurement of composition in the 
region of the knee. As a precursor to this comparison it is useful to see the measurements from the various experiments of the energy spectrum of cosmic rays in this region. These give some information on the systematic differences which may be present between the experiments, since the establishment of an absolute energy scale and the geometrical factor $\left(\mathrm{m}^{2} \mathrm{sr} \mathrm{s}\right)$ of an experiment is often the most demanding task in the overall analysis effort. Figure 8 shows a comparison of the measured all-particle energy spectra from both direct and indirect measurements; only statistical errors are shown. The vertical intensity axis has been multiplied by $E^{2.75}$, to emphasize differences from the overall spectrum of particles. The knee is clearly visible around $3 \mathrm{PeV}$ in the air shower measurements. The overall flux normalization between the direct and indirect measurements is well within the errors, which are large and dominated by the limited geometrical factor of the direct measurements. Any of these measurements typically has a systematic error in the energy scale which is on the order $10-20 \%$, this translates to a $20-40 \%$ shift in the overall intensity values normalization on this plot. The air shower measurements seem to agree almost too well with this level of expected systematic error.

\subsection{Composition estimates}

The derivation of composition from measurements such as those of mean shower maximum $\left\langle X_{\max }\right\rangle$, or derived from the electron and muon numbers, need a choice of the simulation to be used. As shown in Figure 3, both SIBYLL and QGSjet predict an overall logarithmic increase in the depth of shower maximum with energy. Here, to evaluate the size of systematic effects in the simulations, the SIBYLL data are taken from Table 1 [76] and the QGSjet data are from the recent work of Pryke [42] who has calculated similar quantities independently. The slope of this increase is fairly similar, but the overall normalization differences can have an impact on the mass estimates.

As an example we can derive mean logarithmic mass composition from Figure 3 by assuming the mass curves for other nuclei can be derived from a simple logarithmic interpolation between the proton and iron curves shown here. (This means that at any given energy $\langle\log (A)\rangle=\log (56) \times\left(X_{\max }^{p}-\right.$ $\left.\left\langle X_{\max }\right\rangle\right) /\left(X_{\max }^{p}-X_{\max }^{F e}\right)$, where $X_{\max }^{p}$ and $X_{\max }^{F e}$ are the model predictions and $\left\langle X_{\max }\right\rangle$ is the experimentally measured value). Although this procedure is really only accurate if simple superposition is valid, it can give us an approximate basis on which to compare $\langle\log (A)\rangle$ results between experiments. The results of this procedure for the $\left\langle X_{\max }\right\rangle$ results of Figure 3 using QGSjet are shown in Figure 9 where $\langle\log 10(A)\rangle$ is shown versus energy, with the various data sets connected by dashed lines. Also shown here are the results from the direct measurements of the same quantity. There is good agreement between the direct/indirect measurements, although the direct measurements 


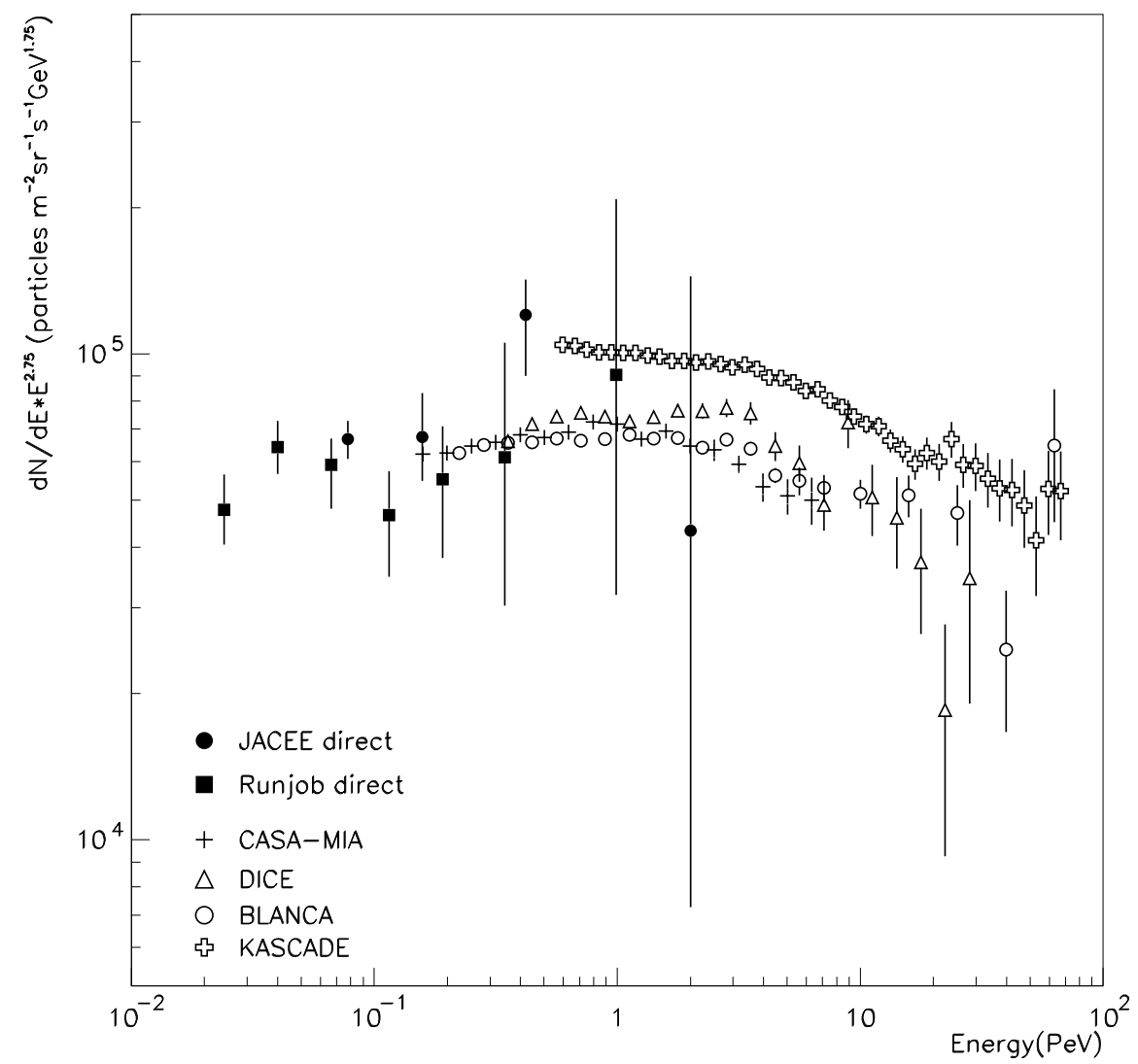

Fig. 8. All-particle energy spectra measurements near the 'knee' from various experiments presented at the workshop

have poor statistics in the knee region.

To evaluate the systematic errors in mass estimate, a similar procedure can be made with the SIBYLL model. The results of this are shown in Figure 10. Here the mean masses are larger, corresponding to the generally deeper shower maxima expected for this model shown in Figure 3. However the general structure of the composition changes is preserved and again these are not inconsistent with the directly measured values.

Within these Cherenkov air shower measurements, all compositions show a decrease in mean mass from around $1 \mathrm{PeV}$ to $3 \mathrm{PeV}$ and two experiments (BLANCA and SPASE-VULCAN) show an increase in mean mass above $3 \mathrm{PeV}$. A systematic shift in the energy scale of any of these air shower experiments will directly affect the inferred mass. The bar labelled 'systematic' at the left hand side of Figures 9 and 10 shows the shift in $\langle\log (A)\rangle$ expected for a $20 \%$ shift in energy scale. This is smaller than the systematic shift introduced between an anaylsis based on QGSjet or SIBYLL 2.1, the two curves shown 


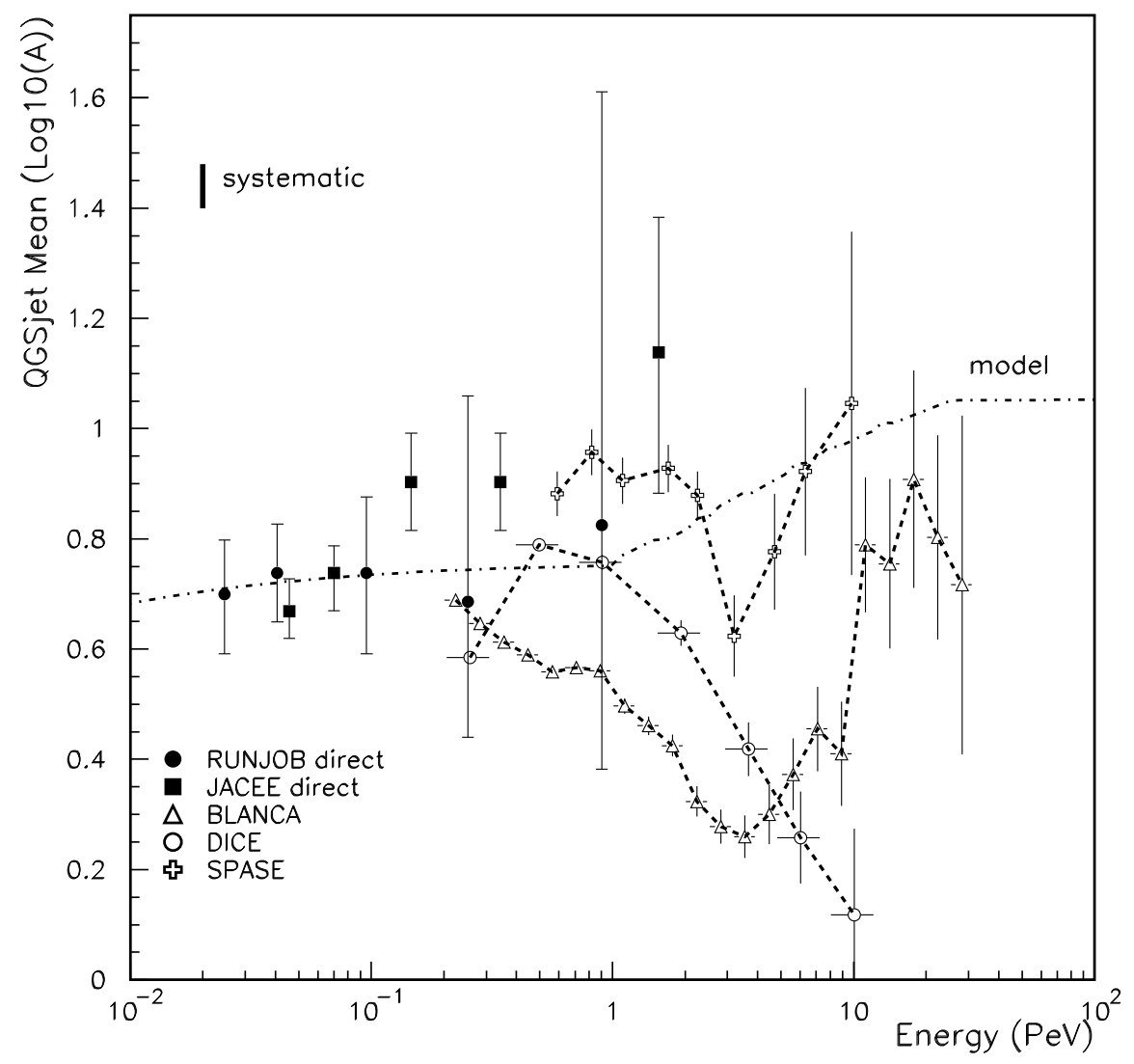

Fig. 9. Results on shower maximum analyzed with the QGSjet model

\section{in Figure 3.}

The goal of composition measurements near the knee is to look for apparent changes in the makeup of the all-particle spectrum which can reveal the origin of this feature. The most basic test which can be made is to examine whether the change in slope at the knee occurs at the same magnetic rigidity for all nuclear species. This would be expected if this feature has an origin either in an intrinsic steepening of the source spectrum of cosmic rays, possibly because diffusive shock acceleration becomes weaker, or because the particles begin to escape from the Galaxy more easily at these energies. An identification of the knee with a simple break in magnetic rigidity would significantly reduce the range of possible origins for this feature.

The signature of a constant magnetic rigidity spectral bend is relatively easy to evaluate in terms of $\langle\log 10(A)\rangle$. Since the all-particle spectrum of cosmic rays measures particle intensities at similar total particle energies $(E=$ $\gamma A m c^{2}, A=$ atomic mass, $m=$ nucleon mass), at the same magnetic rigidity ( $R=\gamma m c A / Z$, since $\beta \approx 1$ at these energies) different nuclear species should 


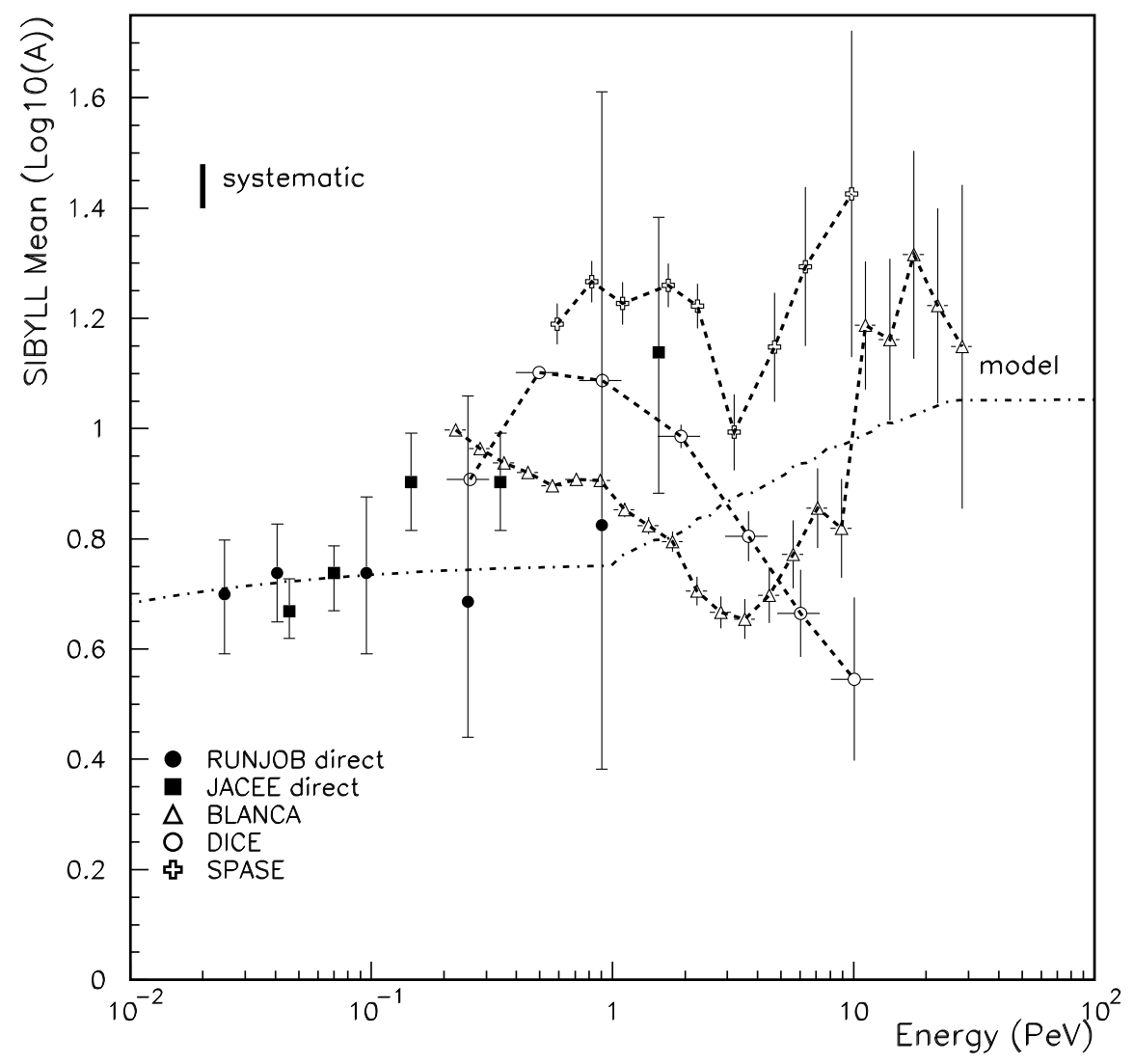

Fig. 10. Results on shower maximum analyzed with the SIBYLL model

show a spectral bend at a total energy $E=Z R c$, where $Z$ is the nuclear charge. For example, if a bend occurs for all nuclei at $R=1 \mathrm{PV}$ this will be observed at $E=1 \mathrm{PeV}$ for protons and at $E=26 \mathrm{PeV}$ for iron nuclei. The heavier nuclei are expected to bend at higher values of $E$, meaning that the mean mass should steadily increase across the knee region.

An estimate of the size of this effect can be made by applying a simple model extrapolating from measured cosmic ray elemental abundances at lower energies. An extension of a model of the Galaxy in which particles have an exponential distribution of pathlengths and an energy dependent leakage probability can be easily made into the 'knee' region [81]. These models produce an equilibrium intensity of particles in the Galaxy which are the result of subjecting a source rigidity spectrum to losses by either spallation in the interstellar medium or escape from the Galactic confinement volume. Introducing a spectral break from $\propto R^{-2.7}$ to $\propto R^{-3.0}$ at $1 \mathrm{PV}$ produces a increase in $\langle\log 10(A)\rangle$ shown by the dot-dash curve labeled model in Figure 9 and Figure 10.

This is a relatively robust prediction since the only assumption made is that 
all nuclear species have the same source magnetic rigidity spectra. The source elemental abundances have been fit to lower energy data [81]. Any model which produces a bend in the rigidity spectra of this size will produce a similar increase in $\langle\log 10(A)\rangle$. The simple model does not agree well with either of the sets of derived mean compositions from the data. The measured values are consistently lighter than expected in two of the experiments. The Cherenkov experiments show a significant tendency to become lighter into the knee region at $3 \mathrm{PeV}$ for all hadronic models. This does not agree with a simple rigidity bend origin for the knee.

Figure 11 shows the estimates of $\langle\log 10(A)\rangle$ derived from CASA-MIA using MOCCA/SIBYLL1.6 and KASCADE using CORSIKA/QGSjet. Also shown are the results from the direct measurements. The simple rigidity bend model is the dot-dashed curve discussed above. The derived compositions agree in general with the direct measurement normalization. The KASCADE data show a trend towards greater mean mass even though there is a dip towards lighter values around $3 \mathrm{PeV}$ similar to that seen with the Cherenkov experiments in Figure 9. CASA-MIA provides the most significant shift to heavy composition of all the experiments discussed here.

\subsection{Multi-Species Fits and Multi-Parameter Correlations}

The discussion in the last section is in terms of the mean value of a parameter, be it $\langle\log 10(A)\rangle$ or $\left\langle X_{\max }\right\rangle$. In fact, although fluctuations are severe, even a simple air shower experiment provides much more information in the form of the distributions and correlations of the observable quantities. This information can be used to improve the quality of the results - for instance providing a fractional composition breakdown into several elemental groups versus energy, rather than simply a mean mass. However, and probably more importantly given the highly indirect nature of the EAS experiments, the information also provides a means to check in detail the consistency of the data with simulations, and hence to search for problems in the shower and detector Monte Carlos.

In the past the limited statistics of the data often meant that distribution analyses were not attempted - this is no longer the case. Additionally full shower and detector simulations, complete with all relevant fluctuations, were not carried out - this is now a standard part of any serious experiment. These two conditions being fullfilled it becomes possible to compare the measured distribution of a composition sensitive parameter to the expected distributions for a range of primary masses. We normally assume that the range of possible primary particles in the knee region is bounded by protons at the light end and iron nuclei at the heavy. If this is the case the measured distribution must 


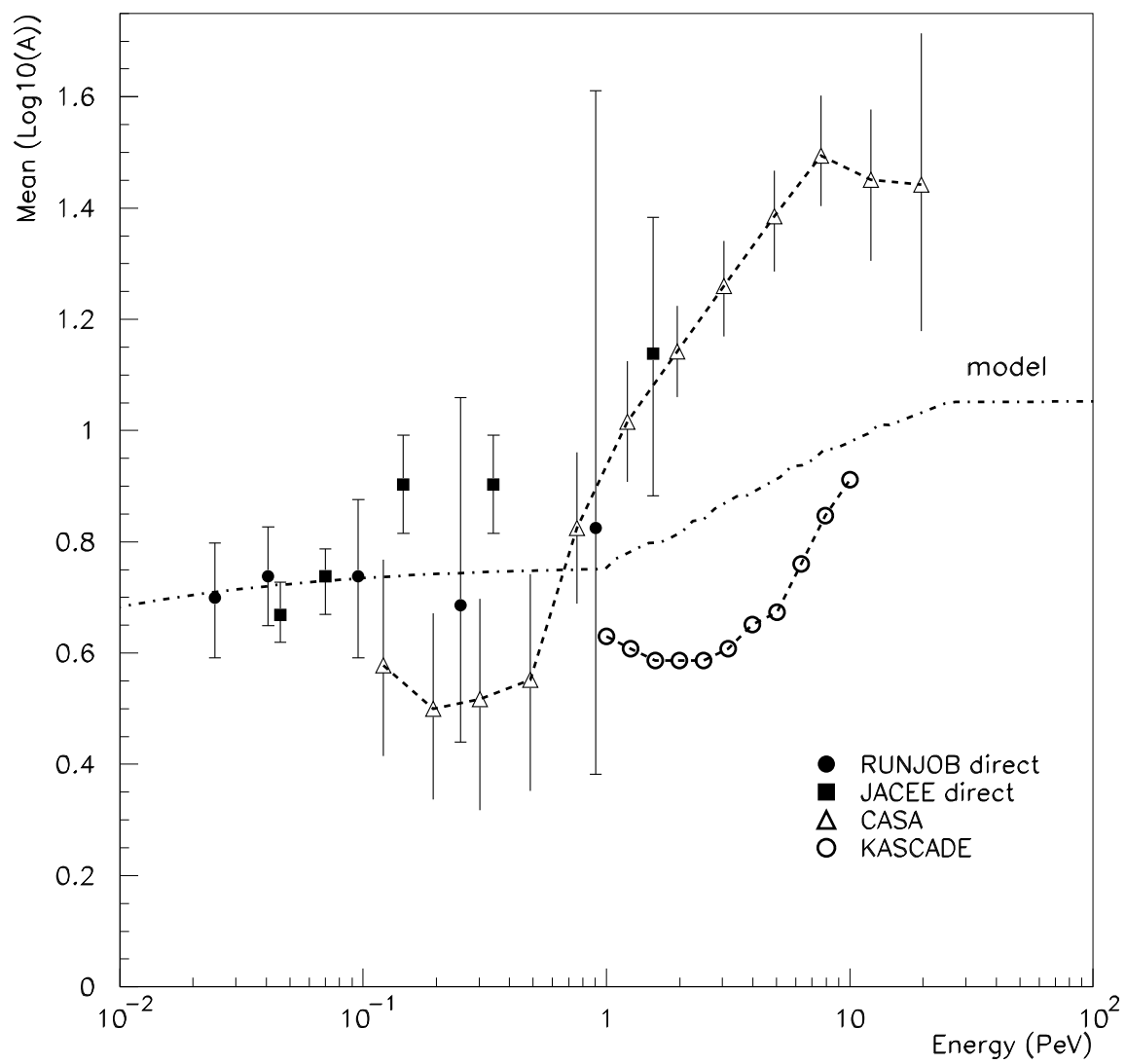

Fig. 11. Mass measurements from KASCADE and CASA-MIA compared with direct measurements.

be reproducible by a sum of simulated distributions for proton, iron and some number of intermediate atomic masses.

It is sometimes claimed that the severe inherent fluctuations of the air shower process limit the number of mass groups that may be fitted. In fact only the statistics of the data and the accuracy of the simulations impose such limits - in principle an arbitrary number of components can be deconvolved. In practice the impact of potential systematic errors in the simulations must be carefully assessed.

If the observed distribution cannot be fit by an appropriate sum of simulated components then either the detector simulation is not adequately reproducing the measurement process, or the shower simulation is flawed. If the shower simulation is suspected then, as discussed in Section 4, by far the most likely culprit is the high energy hadronic interaction model. In this way it can become possible to both choose an appropriate interaction model, and measure the primary composition simultaneously. 

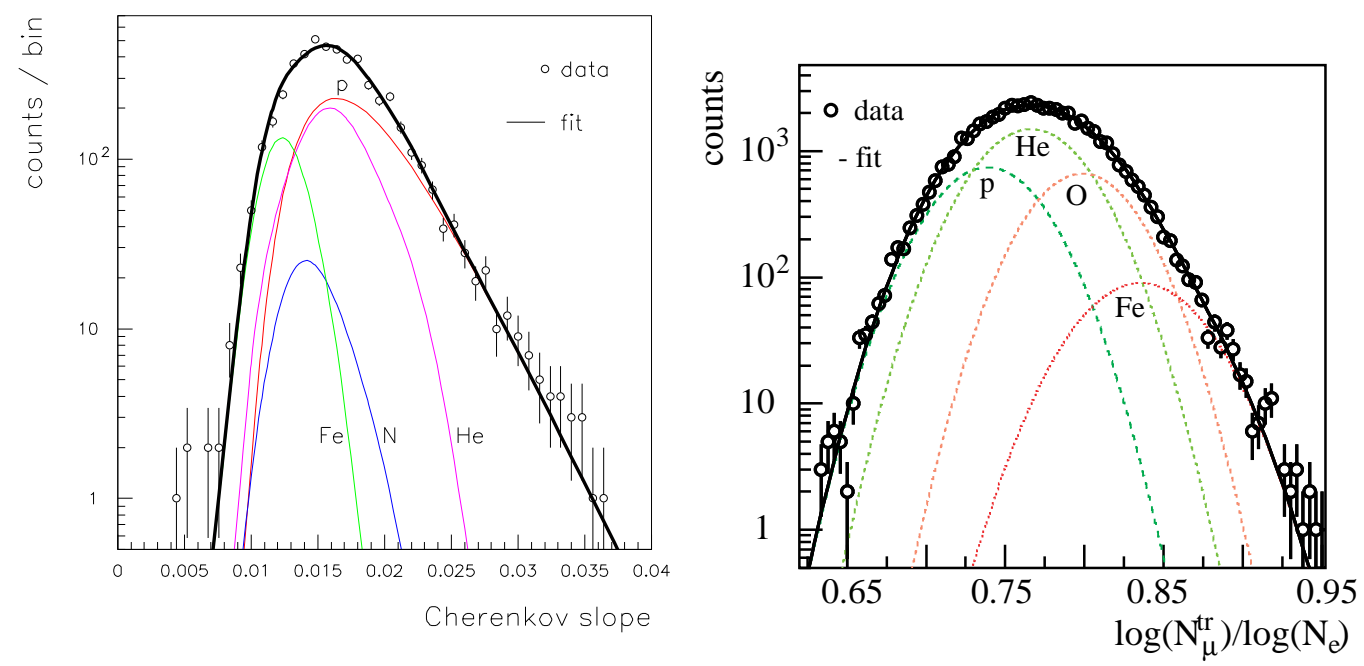

Fig. 12. Two examples of multi-species fits to air shower observables; BLANCA[39] at left, KASCADE[80] at right. See text for details.

Two examples of sucessful multi species fits are shown in Figure 12. Both plots show the distribution of a shower observable which is stongly correlated with primary mass for a group of events in a band of primary energy around $3 \mathrm{PeV}$. At left we have the distribution of the exponential slope of the Cherenkov lateral distribution from BLANCA, and at right the ratio of the truncated muon number to electron number from KASCADE. The binned data is fit to a sum of the expected distributions for four primary species as simulated by CORSIKA-QGSjet, and after processing through the detector simulation, and event reconstruction procedures. The vertical axis is logarithmic in both cases to emphasize the tails of the distribution where inadequate simulations are most likely to be revealed. We can see that both experiments can be well fit by a four species model, and that neither appears to demand a sub-proton or trans-iron component. In fact both data sets demand a four species fit the measured distribution cannot be adequately modeled with fewer (e.g. a simple proton/iron combination). It must be emphasized that such plots are a recent development, and a major step forward in air shower studies.

A trend in recent experiments is to measure multiple parameters of the air shower on an event-by-event basis. It has sometimes been claimed that this can improve the resolution with which primary mass can be measured. However shower fluctuations are so severe that they easily dominate over instrumental resolution for any given parameter in a well designed experiment. Additionally the fluctuations are highly correlated between shower observables - in most cases what one is effectively measuring is the fluctuation of the elasticity (and multiplicity) of the first interaction. The real benefit of multi-parameter measurements is that not only the distributions of the indvidual observables, but also their cross correlations, can be used to compare with simulations. 

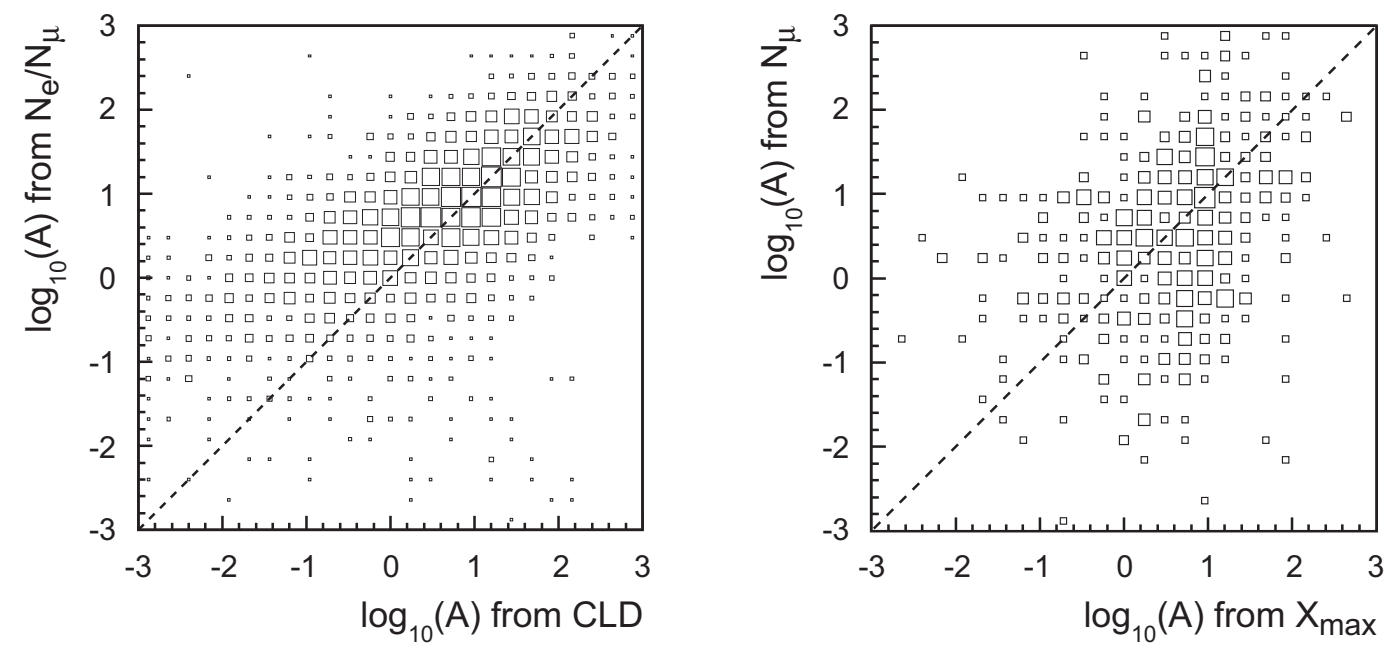

Fig. 13. Two examples of event-by-event correlations between mass estimates near $3 \mathrm{PeV}$ derived from different air shower observables; BLANCA[39] at left from Cherenkov Lateral Distribution and Electron Muon sizes, DICE[37] at right from the Cherenkov image and Muon sizes. See text for details.

Figure 13 shows two examples from the BLANCA and DICE extensions to the CASA-MIA system (see sections 3.6 and 3.5). BLANCA and DICE respectively measure the lateral and longitudinal distributions of Cherenkov light; CASA and MIA simultaneously measure the muon and electron sizes. Both plots are for a band of primary energy around $3 \mathrm{PeV}$ (energy being estimated from the Cherenkov observables). The density of points in the $x y$ plane is shown after making simple parametric transformations from observables to the logarithm of the primary mass $[37,39]$. Simulation plots are very similar for a mixed primary composition. The appearance of points on the figure with severly non-physical atomic masses serves to illustrate that the fluctuations of the air shower process are as large as the difference between the extreme ends of the mass scale which we are trying to measure. Note that the apparently better correlations in the case of BLANCA is at least partly due to the use of $N_{e} / N_{\mu}$ rather than $N_{\mu}$ alone as the mass estimator — most of what is observed is that events with flat Cherenkov lateral distributions have larger electron sizes at ground level.

\section{Conclusions}

The experimental situation in measurements of cosmic-ray composition near the knee of the spectrum has benefited in recent years from several new, relatively large, experiments. In the past these investigations were often undertaken with a few detectors and the simulation power for air showers was limited. Modern experiments have many more channels of information and 
simulation codes have become extensive and sophisticated. In general the experiments discussed here do not suffer from statistical errors of sampling the shower, many measurements are taken for each shower. However the fact remains that trying to determine the identity of the nucleus which initiates an air shower is a very difficult task. A fundamental limit for this task seems to be connected to the accuracy of the air shower models which are used to derive the composition from the data. At present the systematic errors in estimating the absolute energy scale and shifts between the air shower models limit the accuracy in $\langle\log 10(A)\rangle$ to $\sim 0.1$. This is to be compared with an expected shift of $\sim 0.25$ for a simple rigidity bend. There are mixed results from the experiments reported here for the existence of a simple bend. While the ground array measurements show a tendency for a heavier composition with increasing energy, the air cherenkov experiments show a tendency to lighten into the knee region with some increase in mean mass beyond $3 \mathrm{PeV}$.

Several actions could improve this situation in the future. The accuracy of the modelling techniques can be improved if better accelerator results are available in the future as discussed previously. This also includes good measurements for small angle secondaries which at present do not exist. The direct measurements could be improved by longer exposures in space of high energy cosmic ray experiments. In particular this will help with the normalization of systematic errors in energy scale and mean mass measurements with air showers. Plans for using the Space Station for large payloads, such as the ACCESS mission which was discussed at the workshop (see http://access.uchicago.edu), can improve the statistics near $1 \mathrm{PeV}$ by about a factor of 50 over those published here. There are also other possible techniques to measure samples of cosmic rays at high energies. One of these discussed at the meeting was the prospect for measuring the direct emission of Cherenkov light produced by the particle before it interacts in the atmosphere using imaging techniques [82]. This could be a way to get significantly better elemental resolution at high energies with good statistics. Another promising approach is the multi component unfolding analysis discussed in Section 5.3. The KASCADE experiment expects to extend this technique 2 to energies of $10^{18} \mathrm{eV}$.

The workshop would like to thank the Adler Planetarium in Chicago for all their help with organizing this meeting and providing an excellent venue for this event. Additional information discussed at the workshop but not directly presented here can be found at http://knee.uchicago.edu

2 Since this workshop this work has been discussed at the 27th International Cosmic Ray Conference in Hamburg, Germany, 2001. Please see the conference proceedings for details[6]. 


\section{References}

[1] P. O. Lagage and C. J. Cesarsky, Astron. Astrophys., 118, 223 (1983) and 125, 249 (1983).

[2] A.D. Erlykin and A.W. Wolfendale, J. Phys. G, v23, (1997), p979.

[3] E. Juliusson, P. Meyer, and D. Müller, Phys. Rev. Lett. 29 (1972) 447.

[4] F. Arqueros et al., Astron. Astrophys. 359 (2000) 682.

[5] M. Aglietta et al., Astrop. Phys. 10 (1999) 1.

[6] P. Sommers rapporteur report, Proc. 27th Int. Cosmic Ray Conf. (Hamburg) 2001.

[7] R.J. Wilkes et al., Proc 24th Int. Cosmic Ray Conf. (Rome) 2, (1995) 697.

[8] T.H. Burnett et al., Phys. Rev. Lett., 51, (1986) 1010.

[9] T.H. Burnett et al., Ap.J. 349, (1990), 25.

[10] K. Asakamori et al., Proc. 22nd Int. Cosmic Ray Conf. (Dublin), 2, (1991), 97.

[11] K. Asakamori et al., Proc. 23rd Int. Cosmic Ray Conf. (Calgary), 2, (1993), 21 $\& 25$.

[12] E.D. Olsen, PhD thesis, (1995), University of Washington.

[13] K. Asakamori et al., Ap.J. 502 (1998) 278.

[14] M. L. Cherry et al. Proc 26th Int. Cosmic Ray Conf. (Salt Lake City), 3, (1999), 187.

[15] A.V. Apananseko et al., Proc. 26th Int. Cosmic Ray Conf. (Salt Lake City), 3 (1999) 163.

[16] A.V. Apanaseko et al., Astrop. Phys., in press.

[17] N. Hotta et al., Phys. Rev. D, 22 (1980) 1.

[18] M. Okamoto and T. Shibata, Nucl. Instr. and Meth., A257 (1987) 155.

[19] T. Fujinaga et al., Nucl. Instr. and Meth., A276 (1989) 317.

[20] A.V. Apananseko et al., Proc. 26th Int. Cosmic Ray Conf. (Salt Lake City), 3 (1999) 231.

[21] M. Ichimura et al., Phys. Rev. D, 48 (1993) 1949.

[22] H. O. Klages et al., Nucl. Phys. B Proc. Suppl. 52B (1997) 92.

[23] J. Engler et al., Nucl. Instr. Meth., A427 (1999) 528.

[24] T. Antoni et al., Proc. 26th Int. Cosmic Ray Conf. (Salt Lake City), KASCADE collaboration, 15 contributions. 
[25] K.-H. Kampert, FZKA report 6345, Forschungszentrum Karlsruhe 1999.

[26] T. Antoni et al., J. Phys. G: Nucl. Part. Phys. 25 (1999) 2161

[27] T. Antoni et al., accepted by J. Phys. G: Nucl. Part. Phys. 2001.

[28] J.H. Weber et al., Proc. 26th Cosmic Ray Conf., 1 (1999) 341

[29] T. Antoni et al., accepted by Astrop. Phys, 2001.

[30] A. Borione et al., Nucl. Instr. Meth., A346 (1994) 329.

[31] M.A.K. Glasmacher, PhD. thesis (1998) University of Michigan.

[32] M.A.K. Glasmacher et al., Astrop. Phys. 12 (1999) 1.

[33] M.A.K. Glasmacher et al., Astrop. Phys. 10 (1999) 291.

[34] Boothby, K., et al. Proc. 24th Int. Cosmic Ray Conference (Rome), 1995, 2, 697.

[35] Boothby, K., et al. Nucl. Phys. B (Proc. suppl.) 52B, 166.

[36] Boothby, K., et al. Ap. J. Lett., 491, L35-L38, (1997)

[37] S.P. Swordy and D.B. Kieda Astrop. Phys. 13 (2000) 137.

[38] Heck, D., et al. CORSIKA: A Monte Carlo program to Simulate Extensive Air Showers, Forschungszentrum Karlsruhe Report FZKA 6019 (1998)

[39] J.W. Fowler et al. Astrop. Phys. 15 (2001) 49.

[40] J.E. Dickinson and G.M. Spiczak, Nucl. Instr. Meth. A440, 95.

[41] R.S. Fletcher, T.K. Gaisser, P. Lipari, T. Stanev, Phys. Rev. D 50 (1994) 5710 J. Engel, T.K. Gaisser, P. Lipari, T. Stanev, Phys. Rev. D 46 (1992) 5013

[42] C. Pryke, Astrop. Phys., 14 (2001) 319.

[43] W. T. Welford and R. Winston, High Collection Non-imaging Optics, Academic Press, San Diego, 1989.

[44] J. W. Fowler, PhD Thesis, University of Chicago, 2000.

[45] L. F. Fortson et al., Proc. 26th Int. Cosmic Ray Conf., Salt Lake City, 5, 336 (1999).

[46] L. F. Fortson et al., Proc. 26th Int. Cosmic Ray Conf., Salt Lake City, 5, 332 (1999).

[47] Dickinson, J. E. et al. Nucl. Inst. and Meth. A 440, 114 (2000).

[48] Dickinson, J. E. et al. Nucl. Inst. and Meth. A 440, 95 (2000).

[49] Andres E. et al., Astroparticle Phys. 131 (2000).

[50] Karle, A., et al. Astroparticle Phys. 3321 (1995). 
[51] J. R. Patterson and A. M. Hillas, J. Phys. G, 9, 1433 (1983).

[52] A.M. Hillas, Proc. 24th Int. Cosmic Ray Conf. (Rome) 1270 (1995).

[53] Dickinson, J. E. et al. Proc. 26th Int. Cosmic Ray Conf. (Salt Lake City), 3, 136 (1999).

[54] J.A. Hinton, PhD. thesis, University of Leeds (1998).

[55] J. Knapp, D. Heck, G. Schatz, Comparison of Hadronic Interaction Models used in Air Shower Simulations, Forschungszentrum Karlsruhe FZKA 5828 (1996)

[56] D. Heck et al., CORSIKA: A Monte Carlo Code to Simulate Extensive Air Showers, Forschungszentrum Karlsruhe FZKA 6019 (1998)

[57] J. Knapp, D. Heck, Extensive Air Shower Simulation with CORSIKA: A User's Manual, Kernforschungszentrum Karlsruhe KfK 5196 B (1993); for an up to date version see http://www-ik3.fzk.de/ ${ }^{\sim h e c k / c o r s i k a / ~}$

[58] J.N. Capdevielle, J. Phys. G: Nucl. Part. Phys. 15 (1989) 909

[59] K. Werner, Phys. Rep. 232 (1993) 87

[60] N.N. Kalmykov, S.S. Ostapchenko, Yad. Fiz. 56 (1993) 105

N.N. Kalmykov, S.S. Ostapchenko, Phys. At. Nucl. 56 (3) (1993) 346

N.N. Kalmykov, S.S. Ostapchenko, A.I. Pavlov, Bull. Russ. Acad. Sci. (Physics) 58 (1994) 1966

[61] J. Ranft, Phys. Rev. D 51 (1995) 64

[62] H. Fesefeldt, The Simulation of Hadronic Showers -Physics and Application-, Rheinisch-Westfälische Technische Hochschule, Aachen PITHA 85/02 (1985)

[63] V.N. Gribov, Zh. Eksp. Teor. Fiz. 53 (1967) 654 (translated in V. N. Gribov, Sov. Phys. JETP 26 (1968) 414 )

[64] R.J. Glauber, G. Matthiae, Nucl. Phys. B 21 (1970) 135

[65] J. Knapp, Forschungszentrum Karlsruhe FZKA 5970 (1997)

[66] T. Antoni et al., (KASCADE Collaboration), J. Phys. G: Nucl. Part. Phys. 25 (1999) 2161

[67] F. Abe et al., (CDF Collaboration), Phys. Rev. D 50 (1994) 5550

[68] N.A. Amos et al., (E710 Collaboration), Phys. Rev. Lett 68 (1992) 2433

[69] C. Avila et al., (E811 Collaboration), Phys. Lett. B 445 (1999) 419

[70] M. Risse, PhD Thesis University Karlsruhe, Forschungszentrum Karlsruhe FZKA 6493 (2000)

[71] R. Engel, T.K. Gaisser, T. Stanev, Proc. $26^{\text {th }}$ ICRC, Salt Lake City, 1 (1999) 415

R. Engel et al. (in preparation) 
[72] H.J. Drescher, M. Hladik, S. Ostapchenko, K. Werner, hep-ph/9903296

[73] M. Bleicher et al., J. Phys. G: Nucl. Part. Phys. 25 (1999) 1859

[74] G.M. Frichter et al., Phys. Rev. D 50 (1997) 3135

[75] M.M. Block, F. Halzen, T. Stanev, hep-ph/9908222

[76] D. Heck, private communication (2000)

[77] G.J. Alner et al. (UA5 Collaboration), Z. Phys. C 33 (1986) 1

[78] R. Harr et al., Phys. Lett. B 401 (1997) 176

[79] S. Roesler, R Engel, and J. Ranft, Proc. of Int. Conf. on Advanced Monte Carlo for Radiation Physics, Particle Transport Simulation and Applications (MC 2000), Lisbon (2000) (hep-ph/0012252)

[80] K-H Kampert et al., 30th International Symposium on Multiparticle Dynamics, Lake Balaton, Hungary, (2000)

[81] S. Swordy, Proc. 24th Int. Cosmic Ray Conf., Rome, 2, 697 (1995).

[82] D.B. Kieda, S.P. Swordy and S.P. Wakely Astrop, Phys., 15 (2001) 287. 